\title{
DISTRIBUTION OF ZETA ZEROES OF ARTIN-SCHREIER COVERS
}

\author{
Alina Bucur, Chantal David, Brooke Feigon, Matilde Lalín and \\ KANEENIKA Sinha
}

\begin{abstract}
We study the distribution of the zeroes of the zeta functions of the family of Artin-Schreier covers of the projective line over $\mathbb{F}_{q}$ when $q$ is fixed and the genus goes to infinity. We consider both the global and the mesoscopic regimes, proving that when the genus goes to infinity, the number of zeroes with angles in a prescribed non-trivial subinterval of $[-\pi, \pi)$ has a standard Gaussian distribution (when properly normalized).
\end{abstract}

\section{Introduction}

Recently there has been a great deal of interest in statistics for numbers of rational points on curves over finite fields, where the curve varies in a certain family but is always defined over a fixed finite field. This is in contrast to situations studied using Deligne's equidistribution theorem $[5,6]$, which requires the size of the finite field to go to infinity, and which tends to produce statistics related to random matrices in certain monodromy groups. When one fixes the base field, one instead tends to encounter discrete probabilities, typically sums of independent identically distributed random variables. The first result in this direction is the work of Kurlberg and Rudnick for hyperelliptic curves [11]; other cases considered include cyclic $p$-fold covers of the projective line $[2,3]$ (for a slightly different approach see [17]), plane curves [1], complete intersections in projective spaces [4], and general trigonal curves [16].

The number of rational points on a curve over a finite field is determined by the zeta function, and statistical properties of the number of points may be interpreted as properties of the coefficients of the zeta function. A related but somewhat deeper question is to consider statistical properties of zeroes of the zeta function. In the case of hyperelliptic curves, these properties were studied by Faifman and Rudnick [8]. A related family was studied in [18].

In this paper, we make similar considerations for the family of Artin-Schreier covers of $\mathbb{P}^{1}$; this family is interesting because the characteristic of the base field plays a more central role in the definition than in any of the other families mentioned so far. The Artin-Schreier construction is special because it cannot be obtained by base-change from a family of schemes over $\mathbb{Z}$. Since Artin-Schreier covers are cyclic covers of $\mathbb{P}^{1}$, one obtains a direct link between their zeta functions and certain exponential sums; while this is also the case for cyclic $p$-fold covers in characteristics other than $p$, the Artin-Schreier case admits a much more precise analysis. One example of how to

Received by the editors August 24, 2012.

2010 Mathematics Subject Classification. Primary 11G20; Secondary 11M50, 14G15. 
exploit this additional precision is the work of Rojas-Leon and Wan [14] refining the Weil bound for Artin-Schreier curves.

To explain our results in more detail, we introduce some notation. Fix an odd prime $p$ and a finite field $\mathbb{F}_{q}$ of characteristic $p$. Each polynomial $f \in \mathbb{F}_{q}[X]$ whose degree $d$ is not divisible by $p$ defines an Artin-Schreier cover $C_{f}$ of $\mathbb{P}^{1}$ with affine model

$$
Y^{p}-Y=f(X) \text {. }
$$

Since $f$ is a polynomial rather than a more general rational function, $C_{f}$ has $p$-rank 0 . For more details about the structure of the moduli space of Artin-Schreier curves and its $p$-rank strata; see [13]. The Riemann-Hurwitz formula implies that the genus of the above curve is $g=(d-1)(p-1) / 2$. As usual, the Weil zeta function of $C_{f}$ has the form

$$
Z_{C_{f}}(u)=\frac{P_{C_{f}}(u)}{(1-u)(1-q u)} .
$$

Here $P_{C_{f}}(u)$ is a polynomial of degree $2 g=(d-1)(p-1)$, which factors as

$$
P_{C_{f}}(u)=\prod_{\psi \neq 1} L(u, f, \psi)
$$

where the product is taken over the non-trivial additive characters $\psi$ of $\mathbb{F}_{p}$ and $L(u, f, \psi)$ are certain $L$-functions (see (2.1) for the formula). Computing the distribution of the zeroes of the zeta functions $Z_{C_{f}}(u)$ as $C_{f}$ runs over the $\mathbb{F}_{q}$-points of the moduli space $\mathcal{A S}_{g, 0}$ of Artin-Schreier covers of genus $g$ and $p$-rank 0 amounts to computing the distribution of the zeroes of $\prod_{j=1}^{p-1} L\left(u, f, \psi^{j}\right)$ for a fixed non-trivial additive character $\psi$ as $f$ runs over polynomials of degree $d$. In fact, going over each $\mathbb{F}_{q}$-point of the moduli space $\mathcal{A S}_{g, 0}$ once is equivalent to letting $f$ vary over the set $\mathcal{F}_{d}^{\prime}$ of polynomials of degree $d$ containing no non-constant terms of degree divisible by $p$, as such terms can always be eliminated in a unique way without changing the resulting Artin-Schreier cover.

Some statistics for the zeroes in the family of Artin-Schreier covers were considered in the recent work of Entin [7], who employs the methods of Kurlberg and Rudnick [11] to study the variation of the number of points on such a family, then translates the results into information about zeroes. In the present work, we consider the global and mesoscopic regime, as was done by Faifman and Rudnick [8] for the family of hyperelliptic curves.

More precisely, we write

$$
L(u, f, \psi)=\prod_{j=1}^{d-1}\left(1-\alpha_{j}(f, \psi) u\right),
$$

where $\alpha_{j}(f, \psi)=\sqrt{q} \mathrm{e}^{2 \pi \mathrm{i} \theta_{j}(f, \psi)}$ and $\theta_{j}(f, \psi) \in[-1 / 2,1 / 2)$. We study the statistics of the set of angles $\left\{\theta_{j}(f, \psi)\right\}$ as $f$ varies. For an interval $\mathcal{I} \subset[-1 / 2,1 / 2)$, let

$$
\begin{gathered}
N_{\mathcal{I}}(f, \psi):=\#\left\{1 \leq j \leq d-1: \theta_{j}(f, \psi) \in \mathcal{I}\right\}, \\
N_{\mathcal{I}}(f, \psi, \bar{\psi}):=N_{\mathcal{I}}(f, \psi)+N_{\mathcal{I}}(f, \bar{\psi}),
\end{gathered}
$$

and

$$
N_{\mathcal{I}}\left(C_{f}\right):=\sum_{j=1}^{p-1} N_{\mathcal{I}}\left(f, \psi^{j}\right) .
$$


We show that the number of zeroes with angle in a prescribed non-trivial subinterval $\mathcal{I}$ is asymptotic to $2 g|\mathcal{I}|$ (Theorem 4.2), has variance asymptotic to $\frac{2(p-1)}{\pi^{2}} \log (g|\mathcal{I}|$ ) and properly normalized has a Gaussian distribution.

Theorem 1.1. Fix a finite field $\mathbb{F}_{q}$ of characteristic $p$. Let $\mathcal{F}_{d}^{\prime}$ be the family of polynomials defined in (2.2). Then for any real numbers $a<b$ and $0<|\mathcal{I}|<1$ either fixed or $|\mathcal{I}| \rightarrow 0$ while $d|\mathcal{I}| \rightarrow \infty$,

$$
\lim _{d \rightarrow \infty} \operatorname{Prob}_{\mathcal{F}_{d}^{\prime}}\left(a<\frac{N_{\mathcal{I}}\left(C_{f}\right)-(d-1)(p-1)|\mathcal{I}|}{\sqrt{\frac{2(p-1)}{\pi^{2}} \log (d|\mathcal{I}|)}}<b\right)=\frac{1}{\sqrt{2 \pi}} \int_{a}^{b} \mathrm{e}^{-x^{2} / 2} d x .
$$

As noted earlier, this result can also be stated in terms of the $\mathbb{F}_{q}$-points of $\mathcal{A S}_{g, 0}$.

Corollary 1.2. Fix a finite field $\mathbb{F}_{q}$ of characteristic $p$. Then for any real numbers $a<b$ and $0<|\mathcal{I}|<1$ either fixed or $|\mathcal{I}| \rightarrow 0$ while $g|\mathcal{I}| \rightarrow \infty$,

$$
\lim _{g \rightarrow \infty} \operatorname{Prob}_{\mathcal{A S}_{g, 0}\left(\mathbb{F}_{q}\right)}\left(a<\frac{N_{\mathcal{I}}\left(C_{f}\right)-2 g|\mathcal{I}|}{\sqrt{\frac{2(p-1)}{\pi^{2}} \log (g|\mathcal{I}|)}}<b\right)=\frac{1}{\sqrt{2 \pi}} \int_{a}^{b} \mathrm{e}^{-x^{2} / 2} d x .
$$

Theorem 1.1 is obtained by computing the normalized moments of certain approximations of $N_{\mathcal{I}}\left(C_{f}\right)-(p-1)(d-1)|\mathcal{I}|$ given by Beurling-Selberg polynomials to verify that they fit the Gaussian moments. Our results are compatible with the following result for the distribution of zeroes of the $L$-functions $L(u, f, \psi)$ and $L(u, f, \bar{\psi})$.

Proposition 1.3. Fix a finite field $\mathbb{F}_{q}$ of characteristic $p$. Then for any real numbers $a<b$ and $0<|\mathcal{I}|<1$ either fixed or $|\mathcal{I}| \rightarrow 0$ while $d|\mathcal{I}| \rightarrow \infty$,

$$
\lim _{d \rightarrow \infty} \operatorname{Prob}_{\mathcal{F}_{d}^{\prime}}\left(a<\frac{N_{\mathcal{I}}(f, \psi, \bar{\psi})-2(d-1)|\mathcal{I}|}{\sqrt{\frac{4}{\pi^{2}} \log (d|\mathcal{I}|)}}<b\right)=\frac{1}{\sqrt{2 \pi}} \int_{a}^{b} \mathrm{e}^{-x^{2} / 2} d x .
$$

Remark 1.4. Analogous results hold for $N_{\mathcal{I}}(f, \psi)$ as long as the interval $\mathcal{I}$ is symmetric.

Note that Proposition 1.3 is compatible with the philosophy of Katz and Sarnak, which predicts that when $q \rightarrow \infty$, the distribution of $N_{\mathcal{I}}\left(C_{f}\right)$ is the same as the distribution of $\hat{N}_{\mathcal{I}}(U)$, the number of eigenvalues of a $2 g \times 2 g$ matrix $U$ in the monodromy group of $C_{f}$ chosen uniformly at random with respect to the Haar measure. The monodromy groups of Artin-Schreier covers are computed by Katz in $[9,10]$. In the large matrix limit, which corresponds to the limit as $d \rightarrow \infty$ for the family of Artin-Schreier covers because $g=(p-1)(d-1) / 2$, the statistics on $\hat{N}_{\mathcal{I}}(U)$ have been found to have Gaussian fluctuations in various ensembles of random matrices.

1.1. Outline of the paper. This paper is set up as follows. We begin by reviewing basic Artin-Schreier theory in Section 2. In Section 3, we prove two explicit formulas for the zeroes of $L(u, f, \psi)$ which we will need later to compute the moments. In Section 4 , we prove a result about the number of zeroes of the zeta function for a 
fixed Artin-Schreier cover of $\mathbb{P}^{1}$. In Section 5, we recall some facts on Beurling-Selberg polynomials and use them to prove some technical statements about their coefficients. A certain sum of these trigonometric polynomials approximate the characteristic function of the interval $\mathcal{I}$. We use the explicit formula to reduce the problem of studying this sum of Beurling-Selberg polynomials to a problem about sums of characters of traces of a polynomial $f$ evaluated at elements in extensions of $\mathbb{F}_{q}$. In Sections 6 , to 8 we analyze the first, second and third moments of this sum. These moments tell us the expectation and variance of the distribution. In Section 9, we compute the general moments of our approximating function and conclude that it has a standard Gaussian limiting distribution as the degree $d$ of $f$ goes to infinity for $\mathcal{I}$ either fixed or in the mesoscopic regime. Finally, in Section 10, we conclude the proof of Theorem 1.1 by proving that under normalization $N_{\mathcal{I}}\left(C_{f}\right)-(d-1)(p-1)|\mathcal{I}|$ converges in mean square and hence in distribution to our approximating function.

\section{Basic Artin-Schreier theory}

We now recall some more facts about Artin-Schreier covers. For each integer $n \geq 1$, denote by $\operatorname{tr}_{n}: \mathbb{F}_{q^{n}} \rightarrow \mathbb{F}_{p}$ the absolute trace map (not the trace to $\mathbb{F}_{q}$ ). For each polynomial $g \in \mathbb{F}_{q}[X]$ and non-trivial additive character $\psi$ of $\mathbb{F}_{p}$, set

$$
S_{n}(g, \psi)=\sum_{x \in \mathbb{F}_{q^{n}}} \psi\left(\operatorname{tr}_{n}(g(x))\right)
$$

The $L$-functions that appear in (1.2) are given by

$$
L(u, f, \psi)=\exp \left(\sum_{n=1}^{\infty} S_{n}(f, \psi) \frac{u^{n}}{n}\right)=\prod_{P}\left(1-\psi_{f}(P) u^{\operatorname{deg} P}\right)^{-1},
$$

where the product is taken over monic irreducible polynomials in $\mathbb{F}_{q}[X]$. In fact, throughout this paper $P$ will denote such a polynomial and, if $n=\operatorname{deg} P$, we have

$$
\psi_{f}(P)=\sum_{\substack{\alpha \in \mathbb{F}_{q} n \\ P(\alpha)=0}} \psi(f(\alpha))=\psi\left(\operatorname{tr}_{n}(f(\alpha))\right) \text { for any root } \alpha \text { of } P \text {. }
$$

To see that the exponential is equal to the product over primes in (2.1), one has to write the exponential as an Euler product over the closed points of $\mathbb{A}^{1}$. Namely, if we denote by $\mathcal{S}_{n}$ the set of closed points of $\mathbb{A}^{1}$ of degree $n$, we can write

$$
\begin{aligned}
L(u, f, \psi) & =\exp \left(\sum_{n=1}^{\infty} S_{n}(f, \psi) \frac{u^{n}}{n}\right) \\
& =\exp \left(\sum_{n=1}^{\infty} \sum_{x \in \mathcal{S}_{n}} \sum_{k=1}^{\infty} \psi\left(\operatorname{tr}_{k n}(f(x))\right) \frac{u^{k n}}{k}\right)
\end{aligned}
$$


The denominator of the fraction is $k$, not $k n$, because each closed point $x \in \mathcal{S}_{n}$ produces $n$ rational points of $\mathbb{F}_{q^{n}}$. Thus,

$$
\begin{aligned}
L(u, f, \psi) & =\prod_{n=1}^{\infty} \prod_{x \in \mathcal{S}_{n}} \exp \left(\sum_{k=1}^{\infty} \frac{\left(\psi\left(\operatorname{tr}_{n}(f(x))\right) u^{n}\right)^{k}}{k}\right) \\
& =\prod_{n=1}^{\infty} \prod_{x \in \mathcal{S}_{n}}\left(1-\psi\left(\operatorname{tr}_{n}(f(x))\right) u^{n}\right)^{-1} \\
& =\prod_{x \text { closed point of } \mathbb{A}^{1}}\left(1-\psi\left(\operatorname{tr}_{\operatorname{deg} x}(f(x))\right) u^{\operatorname{deg} x}\right)^{-1}
\end{aligned}
$$

which is exactly the product over primes that appears in (2.1).

Note that for the trivial character $\psi=1$, the same formula gives

$$
L(u, f, 1)=Z_{\mathbb{A}^{1}}(u)=\frac{1}{1-q u} .
$$

The factor at infinity is then given by

$$
\psi_{f}(\infty)= \begin{cases}1 & \psi=1 \\ 0 & \psi \neq 1\end{cases}
$$

Therefore we have

$$
Z_{C_{f}}(u)=\prod_{\psi} L^{*}(u, f, \psi)
$$

where $L^{*}(u, f, \psi)$ are the completed $L$-functions,

$$
L^{*}(u, f, \psi)=\prod_{v}\left(1-\psi_{f}\left(P_{v}\right) u^{\operatorname{deg} P_{v}}\right)^{-1} .
$$

Here the product is taken over all places $v$ of $\mathbb{F}_{q}(X)$.

From now on we will fix a non-trivial additive character $\psi$ of $\mathbb{F}_{p}$ given by a certain choice $\zeta$ of a primitive $p$ th root of unity in $\mathbb{C}$. Then, all the other non-trivial characters of $\mathbb{F}_{p}$ are of the form $\sigma \circ \psi$ where $\sigma$ is an automorphism of the cyclotomic field $\mathbb{Q}(\zeta)$. The reciprocals of zeroes of the $L(u, f, \sigma \circ \psi)$ are exactly the Galois conjugates $\sigma\left(\alpha_{j}(f, \psi)\right)$, $1 \leq j \leq d-1$, of the reciprocals of the roots of $L(u, f, \psi)$. In order to compute the distribution of the zeroes of the Weil zeta functions $Z_{C_{f}}$ as $C_{f}$ runs over $\mathcal{A} S_{g, 0}\left(\mathbb{F}_{q}\right)$ we are going to compute the distribution of the angles $\theta_{j}(f, \psi), \theta_{j}(f, \bar{\psi}), 1 \leq j \leq$ $d-1$, for our specific choice of the additive character $\psi$, as $f$ runs through $\mathcal{F}_{d}^{\prime}$, where $g=(d-1)(p-1) / 2$. Since the roots of $L(u, f, \psi)$ and $L(u, f, \bar{\psi})$ are conjugate, it suffices to work with symmetric intervals. The distribution of the roots of the whole zeta function is then obtained by combining the $(p-1) / 2$ distributions for the various choices of $\psi$.

As discussed in the introduction, we will consider $\mathbb{F}_{q}$-points of the moduli space $\mathcal{A S}_{g, 0}$ of Artin-Schreier covers of $p$-rank 0. A cover consists of an Artin-Schreier curve for which we fix an automorphism of order $p$ and an isomorphism between the quotient and $\mathbb{P}^{1}$. We also choose the ramification divisor to be $D=(\infty)$. Thus, the one branch point of our $p$-rank 0 covers is at infinity. 
Concretely, we consider, up to $\mathbb{F}_{q}$-isomorphism, pairs of curves with affine model $C_{f}: Y^{p}-Y=f(X)$ with $f(X)$ a polynomial of degree $d=2 g /(p-1)+1$ not divisible by $p$ together with the automorphism $Y \mapsto Y+1$.

Using the $\mathbb{F}_{q}$-isomorphism $(X, Y) \mapsto\left(X, Y+a X^{k}\right)$, we get that $C_{f}$ is isomorphic to $C_{g}$ where $g(X)=f(X)+a X^{k}-a^{p} X^{k p}$. By using this isomorphism, we are reduced to considering the Artin-Schreier curves with model $C_{f}: Y^{p}-Y=f(X)$ where $f(X)$ is an element of the family $\mathcal{F}_{d}^{\prime}$ defined in the introduction as

$$
\mathcal{F}_{d}^{\prime}=\left\{a_{d} X^{d}+a_{d-1} X^{d-1}+\cdots+a_{0} \in \mathbb{F}_{q}[X]: a_{d} \in \mathbb{F}_{q}^{*}, a_{p k}=0,1 \leq k \leq\left\lfloor\frac{d}{p}\right\rfloor\right\} .
$$

Except for the isomorphisms described above, no two such affine models are isomorphic. Therefore considering all affine models $Y^{p}-Y=f(X)$ with $f(X) \in \mathcal{F}_{d}^{\prime}$ is equivalent to considering all the $\mathbb{F}_{q^{-}}$points of the moduli space $\mathcal{A S}_{g, 0}$. For more details on this one-to-one correspondence between our family and $\mathcal{A S}_{g, 0}\left(\mathbb{F}_{q}\right)$ see [13, Proposition 3.6].

In [7], the author is considering a slightly different family by also allowing twists, i.e., isomorphism over $\mathbb{F}_{q^{p}}$. This amounts to the models $C_{f}: Y^{p}-Y=f(X)$, with $f(X) \in \mathcal{F}_{d}^{\prime \prime}$, where

$$
\mathcal{F}_{d}^{\prime \prime}=\left\{a_{d} X^{d}+a_{d-1} X^{d-1}+\cdots+a_{0} \in \mathbb{F}_{q}[X]: a_{d} \in \mathbb{F}_{q}^{*}, a_{p k}=0,0 \leq k \leq\left\lfloor\frac{d}{p}\right\rfloor\right\} .
$$

Finally, we will denote by

$$
\mathcal{F}_{d}=\left\{a_{d} X^{d}+a_{d-1} X^{d-1}+\cdots+a_{0} \in \mathbb{F}_{q}[X]: a_{d} \in \mathbb{F}_{q}^{*}\right\},
$$

the set of all polynomials of degree $d$ in $\mathbb{F}_{q}[X]$. We will also need the map $\mu: \mathcal{F}_{d} \rightarrow \mathcal{F}_{d}^{\prime}$ defined by

$$
\mu\left(\sum_{i=0}^{d} a_{i} X^{i}\right)=a_{0}+\sum_{\substack{i=1 \\ i \neq k, k \geq 1}}^{d}\left(\sum_{j=0}^{\left\lfloor\log _{p}(d / i)\right\rfloor} a_{i p^{j}}^{p^{-j}}\right) X^{i} .
$$

This map is $\left.q^{\left\lfloor\frac{d}{p}\right.}\right\rfloor$-to-one and preserves the trace of $f(\alpha)$, which will allow us to work with $\mathcal{F}_{d}$ instead of $\mathcal{F}_{d}^{\prime}$ when taking averages.

2.1. Remark on the number of points. For $d$ large enough, the elements of $\mathcal{F}_{d}^{\prime}$ have the same chance as any random polynomial of degree $d$ in $\mathbb{F}_{q}[X]$ to take a given value in some extension of $\mathbb{F}_{q}$. Thus, if $p \nmid n$, as soon as $d-\lfloor d / p\rfloor>q^{n}$, the distribution of $\left\{\# C_{f}\left(\mathbb{F}_{q^{n}}\right): f \in \mathcal{F}_{d}^{\prime}\right\}$ is given by a sum of i.i.d. random variables, one variable for each closed point of $\mathbb{P}^{1}$ of degree $e \mid n$. As long as we stay away from the point at infinity where $f(X)$ has a pole, the fiber above each closed point $x$ of $\mathbb{P}^{1}$ contains pe rational points on the Artin-Schreier cover $C_{f}$ if $x$ happens to be in the kernel of the absolute trace map $\operatorname{tr}_{n}: \mathbb{F}_{q^{n}} \rightarrow \mathbb{F}_{p}$, and no points otherwise. Hence, each random variable in the sum takes the value $p e$ with probability $1 / p$ and 0 with probability $1-1 / p$. The average number of points is then $1+q^{n}$, the constant 1 coming from the point at infinity where the polynomial $f(X)$ has a pole and the fiber above it contains just 1 point. 
If $p \mid n$, the average is higher because there are certain points of $\mathbb{P}^{1}$ of degree $e$ for which the fiber is forced to have pe points, (i.e., the points of degree $e \mid \frac{n}{p}$ ). One adjusts the computation accordingly and obtains that the average number in $C_{f}\left(\mathbb{F}_{q^{n}}\right)$ is now $1+q^{n}+(p-1) q^{n / p}$. This is the essential reason behind Entin's result on the matter [7, Theorem 4], except that his count does not take into account the point at infinity.

\section{Explicit formulas}

Let $K$ be a positive integer, $\mathrm{e}(\theta)=\mathrm{e}^{2 \pi \mathrm{i} \theta}$ and let $h(\theta)=\sum_{|k| \leq K} a_{k} \mathrm{e}(k \theta)$ be a trigonometric polynomial. Then the coefficients $a_{k}$ are given by the Fourier transform

$$
a_{k}=\widehat{h}(k)=\int_{-1 / 2}^{1 / 2} h(\theta) \mathrm{e}(-k \theta) d \theta .
$$

We prove in this section two explicit formulas for $L(u, f, \psi)$, written as an exponential of a sum or as a product over primes as in (2.1). The first explicit formula (Lemma 3.1) will be used to compute the moments over the family $\mathcal{F}_{d}^{\prime}$, and the second explicit formula (Lemma 3.2) will be used to prove a result about the number of zeroes for a fixed $C_{f}$ (see Section 4 ).

Lemma 3.1. Let $h(\theta)=\sum_{|k| \leq K} \widehat{h}(k) \mathrm{e}(k \theta)$ be a trigonometric polynomial. Let $\theta_{j}(f, \psi)$ be the eigenangles of the $L$-function $L(u, f, \psi)$. Then we have

$$
\sum_{j=1}^{d-1} h\left(\theta_{j}(f, \psi)\right)=(d-1) \widehat{h}(0)-\sum_{k=1}^{K} \frac{\widehat{h}(k) S_{k}(f, \psi)+\widehat{h}(-k) S_{k}(f, \bar{\psi})}{q^{k / 2}} .
$$

Proof. Recall from above that

$$
L(u, f, \psi)=\exp \left(\sum_{n=1}^{\infty} S_{n}(f, \psi) \frac{u^{n}}{n}\right)=\prod_{j=1}^{d-1}\left(1-\alpha_{j}(f, \psi) u\right) .
$$

Taking logarithmic derivatives, we have

$$
\frac{d}{d u} \sum_{j=1}^{d-1} \log \left(1-\alpha_{j}(f, \psi) u\right)=\frac{d}{d u} \sum_{n=1}^{\infty} S_{n}(f, \psi) \frac{u^{n}}{n} .
$$

Multiplying both sides by $u$, we obtain

$$
\sum_{j=1}^{d-1} \frac{-\alpha_{j}(f, \psi) u}{1-\alpha_{j}(f, \psi) u}=\sum_{n=1}^{\infty} S_{n}(f, \psi) u^{n}
$$

that is,

$$
-\sum_{j=1}^{d-1} \sum_{n=1}^{\infty}\left(\alpha_{j}(f, \psi) u\right)^{n}=\sum_{n=1}^{\infty} S_{n}(f, \psi) u^{n} .
$$

Comparing coefficients,

$$
-\sum_{j=1}^{d-1}\left(\alpha_{j}(f, \psi)\right)^{n}=S_{n}(f, \psi) .
$$


Thus, for $n>0$, we obtain

$$
-\sum_{j=1}^{d-1} \mathrm{e}^{2 \pi \mathrm{i} n \theta_{j}(f, \psi)}=\frac{S_{n}(f, \psi)}{q^{n / 2}}
$$

For $n<0$, taking complex conjugates, we have by (1.3) and (3.2)

$$
\begin{aligned}
-\sum_{j=1}^{d-1} \mathrm{e}^{2 \pi \mathrm{i} n \theta_{j}(f, \psi)}= & -\overline{\sum_{j=1}^{d-1} \mathrm{e}^{2 \pi i|n| \theta_{j}(f, \psi)}}=-\overline{\sum_{j=1}^{d-1} \frac{\alpha_{j}(f, \psi)^{|n|}}{q^{|n| / 2}}} \\
= & \frac{\overline{S_{|n|}(f, \psi)}}{q^{|n| / 2}}=\frac{S_{|n|}(f, \bar{\psi})}{q^{|n| / 2}}=\frac{S_{|n|}\left(f, \psi^{-1}\right)}{q^{|n| / 2}} .
\end{aligned}
$$

Thus,

$$
\begin{aligned}
\sum_{j=0}^{d-1} h\left(\theta_{j}(f, \psi)\right) & =\sum_{j=1}^{d-1} \sum_{k=-K}^{K} \widehat{h}(k) \mathrm{e}\left(k \theta_{j}(f, \psi)\right) \\
& =(d-1) \widehat{h}(0)+\sum_{j=1}^{d-1} \sum_{k=1}^{K} \widehat{h}(k) \mathrm{e}\left(k \theta_{j}(f, \psi)\right)+\sum_{j=1}^{d-1} \sum_{k=-K}^{-1} \widehat{h}(k) \mathrm{e}\left(k \theta_{j}(f, \psi)\right) \\
& =(d-1) \widehat{h}(0)-\sum_{k=1}^{K} \widehat{h}(k)\left(\frac{S_{k}(f, \psi)}{q^{k / 2}}\right)-\sum_{k=-K}^{-1} \widehat{h}(k)\left(\frac{S_{-k}(f, \bar{\psi})}{q^{-k / 2}}\right) \\
& =(d-1) \widehat{h}(0)-\sum_{k=1}^{K} \frac{\widehat{h}(k) S_{k}(f, \psi)+\widehat{h}(-k) S_{k}(f, \bar{\psi})}{q^{k / 2}}
\end{aligned}
$$

Lemma 3.2. Let $\theta_{j}(f, \psi)$ be the eigenangles of the L-function $L(u, f, \psi)$. Then for any $n \geq 1$,

$$
-\sum_{j=1}^{d-1} \mathrm{e}^{2 \pi \mathrm{i} n \theta_{j}(f, \psi)}=\sum_{\operatorname{deg}(M)=n} \frac{\Lambda(M) \psi_{f}(M)}{q^{n / 2}}
$$

where $M$ runs over monic polynomials in $\mathbb{F}_{q}[X]$,

$$
\Lambda(M)= \begin{cases}\operatorname{deg} P & \text { if } M=P^{k} \text { for some } k \geq 1 \text { and } P \text { irreducible } \\ 0 & \text { otherwise }\end{cases}
$$

and $\psi_{f}\left(P^{k}\right)=\psi_{f}(P)^{k}$.

Proof. Comparing equations (2.1) and (1.3), we have

$$
\prod_{j=1}^{d-1}\left(1-\alpha_{j}(f, \psi) u\right)=\prod_{P}\left(1-\psi_{f}(P) u^{\operatorname{deg} P}\right)^{-1},
$$

where the product on the right-hand side is taken over monic irreducible polynomials in $\mathbb{F}_{q}[X]$. Taking logarithmic derivatives and multiplying by $u$, we deduce that

$$
-\sum_{j=1}^{d-1} \sum_{n=1}^{\infty}\left(\alpha_{j}(f, \psi) u\right)^{n}=\sum_{M} \Lambda(M) u^{\operatorname{deg} M} \psi_{f}(M) .
$$


Comparing the coefficients of $u^{n}$, we obtain

$$
-\sum_{j=1}^{d-1} \alpha_{j}(f, \psi)^{n}=\sum_{\operatorname{deg}(M)=n} \Lambda(M) \psi_{f}(M)
$$

and the result follows by dividing both sides by $q^{n / 2}$.

\section{The distribution of zeroes of $L(u, f, \psi)$}

In this section we use the Erdös-Turán inequality (see [12], Corollary 1.1) to prove a result on the number of eigenangles $\theta_{j}(f, \psi)$ in an interval $\mathcal{I}$ for a fixed $L$-function $L(u, f, \psi)$.

Theorem 4.1 (P. Erdös, P. Turán). Let $x_{1}, x_{2}, \ldots, x_{N}$ be real numbers lying in the unit interval $[-1 / 2,1 / 2)$. For any interval $\mathcal{I} \subseteq[-1 / 2,1 / 2)$, let $A\left(\mathcal{I}, N,\left\{x_{n}\right\}\right)$ denote the number of elements from the above set in $\mathcal{I}$. Let $|\mathcal{I}|$ denote the length of the interval. There exist absolute constants $B_{1}$ and $B_{2}$ such that for any $K \geq 1$,

$$
\left|A\left(\mathcal{I}, N,\left\{x_{n}\right\}\right)-N\right| \mathcal{I}|| \leq \frac{B_{1} N}{K+1}+B_{2} \sum_{k=1}^{K} \frac{1}{k}\left|\sum_{n=1}^{N} \mathrm{e}^{2 \pi \mathrm{i} k x_{n}}\right| .
$$

We now prove the following theorem, which is the analog of Proposition 5.1 in [8].

Theorem 4.2. For any $\mathcal{I} \subseteq[-1 / 2,1 / 2)$, let $N_{\mathcal{I}}(f, \psi):=\#\{1 \leq j \leq d-1$ : $\left.\theta_{j}(f, \psi) \in \mathcal{I}\right\}$. Then

$$
N_{\mathcal{I}}(f, \psi)=(d-1)|\mathcal{I}|+O\left(\frac{d}{\log d}\right) .
$$

Proof. By the Erdös-Turán inequality and Lemma 3.2, we have

$$
\begin{aligned}
\left|N_{\mathcal{I}}(f, \psi)-(d-1)\right| \mathcal{I}|| & \ll \frac{d}{K}+\sum_{k=1}^{K} \frac{1}{k}\left|\sum_{\operatorname{deg} M=k} \frac{\Lambda(M) \psi_{f}(M)}{q^{k / 2}}\right| \\
& \ll \frac{d}{K}+\sum_{k=1}^{K} \frac{1}{q^{k / 2}} \sum_{\substack{M=P a, a \geq 1 \\
\operatorname{deg} M=k}} 1 .
\end{aligned}
$$

Applying the function-field analog of the prime number theorem, the above expression is $\ll \frac{d}{K}+\frac{q^{K / 2}}{K}$. Choosing $K=\left[\frac{\log d}{\log q}\right]$, we deduce the theorem.

\section{Beurling-Selberg functions}

By the functional equation, the conjugate of a root of $Z_{C_{f}}(u)$ is also a root so we can restrict to considering symmetric intervals. Let $0<\beta<1$ and set $\mathcal{I}=[-\beta / 2, \beta / 2] \subset$ $[-1 / 2,1 / 2)$. We are going to approximate the characteristic function of $\mathcal{I}$, $\chi_{\mathcal{I}}$, with Beurling-Selberg polynomials $I_{K}^{ \pm}$. We will use the following properties of the coefficients of Beurling-Selberg polynomials (see [12], ch 1.2). 
(a) The $I_{K}^{ \pm}$are trigonometric polynomials of degree $\leq K$, i.e.,

$$
I_{K}^{ \pm}(x)=\sum_{|k| \leq K} \widehat{I}_{K}^{ \pm}(k) \mathrm{e}(k x) .
$$

(b) The Beurling-Selberg polynomials bound the characteristic function from below and above:

$$
I_{K}^{-} \leq \chi_{\mathcal{I}} \leq I_{K}^{+}
$$

(c) The integral of Beurling-Selberg polynomials is close to the length of the interval:

$$
\int_{-1 / 2}^{1 / 2} I_{K}^{ \pm}(x) d x=\int_{-1 / 2}^{1 / 2} \chi_{\mathcal{I}}(x) d x \pm \frac{1}{K+1} .
$$

(d) The $I_{K}^{ \pm}$are even (since we are taking the interval $\mathcal{I}$ to be symmetric about the origin). It then follows that the Fourier coefficients are also even, i.e., $\widehat{I}_{K}^{ \pm}(-k)=\widehat{I}_{K}^{ \pm}(k)$ for $|k| \leq K$.

(e) The non-zero Fourier coefficients are also close to those of the characteristic function:

$$
\left|\widehat{I}_{K}^{ \pm}(k)-\widehat{\chi}_{\mathcal{I}}(k)\right| \leq \frac{1}{K+1} \quad \Longrightarrow \quad \widehat{I}_{K}^{ \pm}(k)=\frac{\sin (\pi k|\mathcal{I}|)}{\pi k}+O\left(\frac{1}{K+1}\right), \quad k \geq 1 .
$$

This implies the following bound:

$$
\left|\widehat{I}_{K}^{ \pm}(k)\right| \leq \frac{1}{K+1}+\min \left\{|\mathcal{I}|, \frac{\pi}{|k|}\right\}, \quad 0<|k| \leq K .
$$

Proposition 5.1 (Proposition 4.1, [8]). For $K \geq 1$ such that $K|\mathcal{I}|>1$, we have

$$
\begin{aligned}
\sum_{k \geq 1} \widehat{I}_{K}^{ \pm}(2 k) & =O(1), \\
\sum_{k \geq 1} \widehat{I}_{K}^{ \pm}(k)^{2} k & =\frac{1}{2 \pi^{2}} \log (K|\mathcal{I}|)+O(1), \\
\sum_{k \geq 1} \widehat{I}_{K}^{+}(k) \widehat{I}_{K}^{-}(k) k & =\frac{1}{2 \pi^{2}} \log (K|\mathcal{I}|)+O(1) .
\end{aligned}
$$

Note that for a given $K$ these sums are actually finite, since the Beurling-Selberg polynomials $I_{K}^{ \pm}$have degree at most $K$.

Proof. The first two statements are proven in Proposition 4.1 of [8]. Since

$$
\widehat{I}_{K}^{ \pm}(k)=\frac{\sin (\pi k|\mathcal{I}|)}{\pi k}+O\left(\frac{1}{K}\right),
$$

holds for both $\widehat{I}_{K}^{+}(k)$ and $\widehat{I}_{K}^{-}(k)$, the third statement follows by exactly the same proof as the second statement.

We will also need the following estimates.

Proposition 5.2. For $\alpha_{1}, \ldots, \alpha_{r}, \gamma_{1}, \ldots, \gamma_{r}>0$, and $\beta_{1}, \ldots, \beta_{r} \in \mathbb{R}$, we have,

$$
\sum_{k_{1}, \ldots, k_{r} \geq 1} \widehat{I}_{K}^{ \pm}\left(k_{1}\right)^{\alpha_{1}} \cdots \widehat{I}_{K}^{ \pm}\left(k_{r}\right)^{\alpha_{r}} k_{1}^{\beta_{1}} \cdots k_{r}^{\beta_{r}} q^{-\gamma_{1} k_{1}-\cdots-\gamma_{r} k_{r}}=O(1) .
$$


For $\alpha_{1}, \alpha_{2}, \gamma>0$, and $\beta \in \mathbb{R}$,

$$
\sum_{k \geq 1} \widehat{I}_{K}^{ \pm}(k)^{\alpha_{1}} \widehat{I}_{K}^{ \pm}(2 k)^{\alpha_{2}} k^{\beta} q^{-\gamma k}=O(1) .
$$

Proof. Since $\left|\widehat{I}_{K}^{ \pm}(k)\right| \leq \frac{1}{K+1}+\min \left\{|\mathcal{I}|, \frac{\pi}{|k|}\right\}$, we obtain

$$
\begin{aligned}
& \left|\sum_{k_{1}, \ldots, k_{r} \geq 1} \widehat{I}_{K}^{ \pm}\left(k_{1}\right)^{\alpha_{1}} \cdots \widehat{I}_{K}^{ \pm}\left(k_{r}\right)^{\alpha_{r}} k_{1}^{\beta_{1}} \cdots k_{r}^{\beta_{r}} q^{-\gamma_{1} k_{1}-\cdots-\gamma_{r} k_{r}}\right| \\
& \quad \ll \sum_{k_{1}, \ldots, k_{r} \geq 1} k_{1}^{\beta_{1}} \cdots k_{r}^{\beta_{r}} q^{-\gamma_{1} k_{1}-\cdots-\gamma_{r} k_{r}} .
\end{aligned}
$$

Since $\sum_{k \geq 1} k^{\beta} q^{-\gamma k}=O(1)$ for $q>1$ and $\gamma>0$, we obtain that the right-hand side above is also equal to $O(1)$. The second equation is a particular form of the more general equation established above.

\section{First moment}

Recall that $N_{\mathcal{I}}(f, \psi)$ denotes the number of angles $\theta_{j}(f, \psi)$ of the zeroes of the $L$-function $L(u, f, \psi)$ in the interval $\mathcal{I} \subset[-1 / 2,1 / 2)$ of length $0<|\mathcal{I}|<1$.

From now on, for a function $\phi: \mathcal{F}_{d}^{\prime} \rightarrow \mathbb{C}$, we denote its average by

$$
\langle\phi(f)\rangle:=\frac{1}{\left|\mathcal{F}_{d}^{\prime}\right|} \sum_{f \in \mathcal{F}_{d}^{\prime}} \phi(f) .
$$

We want to compute the first moment

$$
\left\langle N_{\mathcal{I}}(f, \psi)\right\rangle=\frac{1}{\left|\mathcal{F}_{d}^{\prime}\right|} \sum_{f \in \mathcal{F}_{d}^{\prime}} N_{\mathcal{I}}(f, \psi) .
$$

We will do so by proving the following result.

Theorem 6.1. As $d \rightarrow \infty$,

$$
\left\langle N_{\mathcal{I}}(f, \psi)-(d-1)|\mathcal{I}|\right\rangle=O(1) .
$$

Remark 6.2. Recall that in Theorem 4.2 we showed that

$$
N_{\mathcal{I}}(f, \psi)-(d-1)|\mathcal{I}|=O\left(\frac{d}{\log d}\right) .
$$

Theorem 6.1, on the other hand, gives us a far better estimate for the average of $\left\langle N_{\mathcal{I}}(f, \psi)-(d-1)|\mathcal{I}|\right\rangle$ than we could have derived from Theorem 4.2.

For the proof of Theorem 6.1, we will use the Beurling-Selberg approximation of the characteristic function of the interval $\mathcal{I}$. By property (b) of the Beurling-Selberg polynomials,

$$
\sum_{j=1}^{d-1} I_{K}^{-}\left(\theta_{j}(f, \psi)\right) \leq N_{\mathcal{I}}(f, \psi) \leq \sum_{j=1}^{d-1} I_{K}^{+}\left(\theta_{j}(f, \psi)\right) .
$$


With the explicit formula of Lemma 3.1 and property (c), we write

$$
\sum_{j=1}^{d-1} I_{K}^{ \pm}\left(\theta_{j}(f, \psi)\right)=(d-1)|\mathcal{I}|-S^{ \pm}(K, f, \psi) \pm \frac{d-1}{K+1}
$$

where

$$
S^{ \pm}(K, f, \psi):=\sum_{k=1}^{K} \frac{\widehat{I}_{K}^{ \pm}(k) S_{k}(f, \psi)+\widehat{I}_{K}^{ \pm}(-k) S_{k}(f, \bar{\psi})}{q^{k / 2}} .
$$

This gives

$$
-S^{-}(K, f, \psi)-\frac{d-1}{K+1} \leq N_{\mathcal{I}}(f, \psi)-(d-1)|\mathcal{I}| \leq-S^{+}(K, f, \psi)+\frac{d-1}{K+1} .
$$

In order to complete the proof it remains to estimate $\left\langle S^{ \pm}(K, f, \psi)\right\rangle$. We will need the following results from [7]. As we remarked in Section 2, we are using a slightly different description for the family of Artin-Schreier covers since we do not allow twists. Because of that, our results are slightly simpler than those stated in [7]. We have also modified the original notation, so that it fits the generalization that we pursue in the next sections.

Lemma 6.3 ([7], Lemma 5.2). Let $h$ be an integer, $p \nmid h$. Assume $k<d$ and $\alpha \in \mathbb{F}_{q^{k}}$. Then

$$
\left\langle h \psi\left(\operatorname{tr}_{k} f(\alpha)\right)\right\rangle= \begin{cases}1 & p \mid k, \alpha \in \mathbb{F}_{q^{k / p}} \\ 0 & \text { otherwise. }\end{cases}
$$

Proof. If $p \mid k$ and $\alpha \in \mathbb{F}_{q^{k / p}}$ then $\operatorname{tr}_{k}(f(\alpha))=p \operatorname{tr}_{\frac{k}{p}}(f(\alpha))=0$ so $\left\langle\psi\left(\operatorname{tr}_{k} f(\alpha)\right)\right\rangle=1$. For the remaining case, we first note that the average is the same if we average over the family $\mathcal{F}_{d}$ of degree $d$ polynomials (without the condition $a_{p k}=0$ ). This is due to the existence of the map $\mu$ defined by $(2.3)$.

Denote by $u$ the degree of $\alpha$ over $\mathbb{F}_{q}$. Since $u \leq k<d$ the map

$$
\tau: \mathcal{F}_{d} \rightarrow \mathbb{F}_{q^{u}}
$$

defined by $\tau(f)=f(\alpha)$ is $(q-1) q^{d-u}$-to-one. Thus as $f$ ranges over $\mathcal{F}_{d}, f(\alpha)$ takes each value in $\mathbb{F}_{q^{u}}$ an equal number of times. Since $p \nmid \frac{k}{u}, \operatorname{tr}_{k}(f(\alpha))=\frac{k}{u} \operatorname{tr}_{u}(f(\alpha))$ also takes every value in $\mathbb{F}_{p}$ the same number of times as $f$ ranges over $\mathcal{F}_{d}$ and the same is true for $h \operatorname{tr}_{k}(f(\alpha))$. Thus each $p$ th root of unity occurs the same number of times in $\psi\left(h \operatorname{tr}_{k}(f(\alpha))\right)$ as $f$ ranges over $\mathcal{F}_{d}$ and so the average is 0 .

The lemma has the following consequence.

Corollary 6.4 ([7], Corollary 5.3). Let $h$ be an integer, $p \nmid h$. Assume $k<d$ and set

$$
M_{1, d}^{k, 1, h}:=\left\langle q^{-k / 2} \sum_{\alpha \in \mathbb{F}_{q^{k}}} \psi\left(h \operatorname{tr}_{k} f(\alpha)\right)\right\rangle .
$$

Then

$$
M_{1, d}^{k, 1, h}=e_{p, k} q^{-(1 / 2-1 / p) k},
$$


where

$$
e_{p, k}= \begin{cases}0 & p \nmid k \\ 1 & p \mid k\end{cases}
$$

We also denote

$$
M_{1, d}^{k,-1, h}:=\left\langle q^{-k / 2} \sum_{\alpha \in \mathbb{F}_{q^{k}}} \psi\left(-h \operatorname{tr}_{k} f(\alpha)\right)\right\rangle .
$$

Clearly, $M_{1, d}^{k,-1, h}=\overline{M_{1, d}^{k, 1, h}}$.

Note that changing $h$ allows us to vary the character from $\psi$ to $\psi^{h}$. This will be useful later.

Proof (Theorem 6.1). We have that

$$
\begin{aligned}
\left\langle S^{ \pm}(K, f, \psi)\right\rangle & =\sum_{k=1}^{K} \frac{\widehat{I}_{K}^{ \pm}(k)\left\langle S_{k}(f, \psi)\right\rangle+\widehat{I}_{K}^{ \pm}(-k)\left\langle S_{k}(f, \bar{\psi})\right\rangle}{q^{k / 2}} \\
& =\sum_{k=1}^{K} \widehat{I}_{K}^{ \pm}(k) M_{1, d}^{k, 1,1}+\widehat{I}_{K}^{ \pm}(-k) M_{1, d}^{k,-1,1} \\
& =2 \sum_{k=1}^{K} \widehat{I}_{K}^{ \pm}(k) e_{p, k} q^{-(1 / 2-1 / p) k}
\end{aligned}
$$

and the result follows from property (e) and (6.2) taking $K=c d$ with $c<1$.

Remark 6.5. We denote by

$$
C(K):=\sum_{k=1}^{K} \widehat{I}_{K}^{ \pm}(k) e_{p, k} q^{-(1 / 2-1 / p) k}
$$

and

$$
C:=\sum_{k=1}^{\infty} \frac{\sin (\pi k|\mathcal{I}|)}{\pi k} e_{p, k} q^{-(1 / 2-1 / p) k} .
$$

These terms will reappear in the computation of the higher moments. Note that, since $p>2$, the above infinite series converges absolutely. By Proposition 5.2, $C(K)=O(1)$. By property (e) of the Beurling-Selberg polynomials, $C=C(K)+O(1 / K)$.

\section{Second moment}

Let

$$
S^{ \pm}\left(K, C_{f}\right)=\sum_{h=1}^{p-1} S^{ \pm}\left(K, f, \psi^{h}\right),
$$

where $S^{ \pm}(K, f, \psi)$ is defined in (6.1). 
In the next sections, we are computing the moments of $S^{ \pm}\left(K, C_{f}\right)$. We show that they fit the Gaussian moments when properly normalized (Theorem 9.7). We will then use this result to show that

$$
\frac{N_{\mathcal{I}}\left(C_{f}\right)-(p-1)(d-1)|\mathcal{I}|}{\sqrt{\frac{2(p-1)}{\pi^{2}} \log (d|\mathcal{I}|)}}
$$

converges to a normal distribution as $d \rightarrow \infty$, since it converges in mean square to

$$
\frac{S^{ \pm}\left(K, C_{f}\right)}{\sqrt{\frac{2(p-1)}{\pi^{2}} \log (d|\mathcal{I}|)}} .
$$

The following lemma is a generalization of Lemma 6.2 in [7], that also takes into account the difference in our family of Artin-Schreier covers.

Recall that $\psi^{j}(\alpha)=\psi(j \alpha)$ for $\alpha \in \mathbb{F}_{p}$. We have the following

Lemma 7.1. Fix $h_{1}, h_{2}$ such that $p \nmid h_{1} h_{2}$ and let $e_{1}, e_{2} \in\{-1,1\}$. Assume $k_{1}, k_{2}>0$, $k_{1}+k_{2}<d$. Let $\alpha_{1} \in \mathbb{F}_{q^{k_{1}}}, \alpha_{2} \in \mathbb{F}_{q^{k_{2}}}$ with monic minimal polynomials $g_{1}, g_{2}$ of degrees $u_{1}, u_{2}$ over $\mathbb{F}_{q}$, respectively. We have

$$
\left\langle\psi\left(e_{1} h_{1} \operatorname{tr}_{k_{1}} f\left(\alpha_{1}\right)+e_{2} h_{2} \operatorname{tr}_{k_{2}} f\left(\alpha_{2}\right)\right)\right\rangle= \begin{cases}1, & g_{1}=g_{2}, p \mid \frac{e_{1} h_{1} k_{1}+e_{2} h_{2} k_{2}}{u_{1}}, p \nmid \frac{k_{1} k_{2}}{u_{1} u_{2}} \\ & \text { or } p \mid\left(\frac{k_{1}}{u_{1}}, \frac{k_{2}}{u_{2}}\right) ; \\ 0, & \text { otherwise. }\end{cases}
$$

Proof. If $p \mid \frac{k_{2}}{u_{2}}$ then $\operatorname{tr}_{k_{2}} f\left(\alpha_{2}\right)=p \operatorname{tr}_{\frac{k_{2}}{p}} f\left(\alpha_{2}\right)=0$, so

$$
\left\langle\psi\left(e_{1} h_{1} \operatorname{tr}_{k_{1}} f\left(\alpha_{1}\right)+e_{2} h_{2} \operatorname{tr}_{k_{2}} f\left(\alpha_{2}\right)\right)\right\rangle=\left\langle\psi\left(e_{1} h_{1} \operatorname{tr}_{k_{1}} f\left(\alpha_{1}\right)\right)\right\rangle .
$$

By Lemma 6.3, this equals 0 if $p \nmid \frac{k_{1}}{u_{1}}$ and 1 if $p \mid \frac{k_{1}}{u_{1}}$ as $p \nmid e_{1} h_{1}$.

The only remaining case is when $p \nmid \frac{k_{1} k_{2}}{u_{1} u_{2}}$. We first suppose that $g_{1} \neq g_{2}$. We note that we will have the same value if we average over $\mathcal{F}_{d}$ rather than $\mathcal{F}_{d}^{\prime}$ due to the existence of the map $\mu$ defined by (2.3). Since $u_{1}+u_{2} \leq k_{1}+k_{2}<d$, the map

$$
\tau: \mathcal{F}_{d} \rightarrow \mathbb{F}_{q}[X] /\left(g_{1} g_{2}\right) \simeq \mathbb{F}_{q^{u_{1}}} \times \mathbb{F}_{q^{u_{2}}}
$$

is exactly $(q-1) q^{d-u_{1}-u_{2}}$-to-one. Hence, as $f$ ranges over $\mathcal{F}_{d},\left(f\left(\alpha_{1}\right), f\left(\alpha_{2}\right)\right)$ takes every value in $\mathbb{F}_{q^{u_{1}}} \times \mathbb{F}_{q^{u_{2}}}$ the same number of times. Now, since $p \nmid \frac{e_{1} h_{1} k_{1}}{u_{1}}$ and $p \nmid \frac{e_{2} h_{2} k_{2}}{u_{2}}$,

$$
\left(\operatorname{tr}_{k_{1}} f\left(\alpha_{1}\right), \operatorname{tr}_{k_{2}} f\left(\alpha_{2}\right)\right)=\left(\frac{e_{1} h_{1} k_{1}}{u_{1}} \operatorname{tr}_{u_{1}}\left(f\left(\alpha_{1}\right)\right), \frac{e_{2} h_{2} k_{2}}{u_{2}} \operatorname{tr}_{u_{2}}\left(f\left(\alpha_{2}\right)\right)\right)
$$

also takes every value in $\mathbb{F}_{p} \times \mathbb{F}_{p}$ the same number of times as $f$ ranges over $\mathcal{F}_{d}$. Then

$$
\begin{aligned}
& \psi\left(e_{1} h_{1} \operatorname{tr}_{k_{1}}\left(f\left(\alpha_{1}\right)\right)+e_{2} h_{2} \operatorname{tr}_{k_{2}}\left(f\left(\alpha_{2}\right)\right)\right) \\
& \quad=\psi\left(e_{1} h_{1} \frac{k_{1}}{u_{1}} \operatorname{tr}_{u_{1}}\left(f\left(\alpha_{1}\right)\right)+e_{2} h_{2} \frac{k_{2}}{u_{2}} \operatorname{tr}_{u_{2}}\left(f\left(\alpha_{2}\right)\right)\right)
\end{aligned}
$$

assumes every $p$ th root of unity equally many times as we average over $\mathcal{F}_{d}$ and so the average is 0 . 
If $g_{1}=g_{2}$, then $\alpha_{1}$ and $\alpha_{2}$ are conjugates over $\mathbb{F}_{q}$ and so are $f\left(\alpha_{1}\right)$ and $f\left(\alpha_{2}\right)$. Then $\operatorname{tr}_{u_{1}} f\left(\alpha_{1}\right)=\operatorname{tr}_{u_{1}} f\left(\alpha_{2}\right)$. This implies

$$
\begin{aligned}
e_{1} h_{1} \operatorname{tr}_{k_{1}} f\left(\alpha_{1}\right)+e_{2} h_{2} \operatorname{tr}_{k_{2}} f\left(\alpha_{2}\right) & =e_{1} h_{1} \frac{k_{1}}{u_{1}} \operatorname{tr}_{u_{1}} f\left(\alpha_{1}\right)+e_{2} h_{2} \frac{k_{2}}{u_{1}} \operatorname{tr}_{u_{1}} f\left(\alpha_{2}\right) \\
& =\frac{e_{1} h_{1} k_{1}+e_{2} h_{2} k_{2}}{u_{1}} \operatorname{tr}_{u_{1}} f\left(\alpha_{1}\right)
\end{aligned}
$$

which is zero when $p \mid \frac{e_{1} h_{1} k_{1}+e_{2} h_{2} k_{2}}{u_{1}}$. If $p$ does not divide $\frac{e_{1} h_{1} k_{1}+e_{2} h_{2} k_{2}}{u_{1}}$ then

$$
\left\langle\psi\left(e_{1} h_{1} \operatorname{tr}_{k_{1}} f\left(\alpha_{1}\right)+e_{2} h_{2} \operatorname{tr}_{k_{2}} f\left(\alpha_{2}\right)\right)\right\rangle=\left\langle\psi\left(\frac{e_{1} h_{1} k_{1}+e_{2} h_{2} k_{2}}{u_{1}} \operatorname{tr}_{u_{1}} f\left(\alpha_{1}\right)\right)\right\rangle=0
$$

by Lemma 6.3 .

For positive integers $k_{1}, k_{2}, h_{1}, h_{2}$ with $p \nmid h_{1} h_{2}$ and $e_{1}, e_{2} \in\{-1,1\}$, let

$$
\begin{aligned}
M_{2, d}^{\left(k_{1}, k_{2}\right),\left(e_{1}, e_{2}\right),\left(h_{1}, h_{2}\right)}: & =\left\langle q^{-\left(k_{1}+k_{2}\right) / 2} \sum_{\substack{\alpha_{1} \in \mathbb{F}_{q^{k}} k_{1} \\
\alpha_{2} \in \mathbb{F}_{q^{k}}}} \psi\left(e_{1} h_{1} \operatorname{tr}_{k_{1}} f\left(\alpha_{1}\right)+e_{2} h_{2} \operatorname{tr}_{k_{2}} f\left(\alpha_{2}\right)\right)\right\rangle \\
& =q^{-\left(k_{1}+k_{2}\right) / 2} \sum_{\substack{\alpha_{1} \in \mathbb{F} \\
\alpha_{2} \in \mathbb{F}^{k}}}\left\langle\psi\left(e_{1} h_{1} \operatorname{tr}_{k_{1}} f\left(\alpha_{1}\right)+e_{2} h_{2} \operatorname{tr}_{k_{2}} f\left(\alpha_{2}\right)\right)\right\rangle .
\end{aligned}
$$

Then, we have the following analog of Theorem 8 in [7].

Theorem 7.2. Assume $k_{1} \geq k_{2}>0$ and $k_{1}+k_{2}<d$. Let $0<h_{1}, h_{2} \leq(p-1) / 2$. Then

$$
\begin{aligned}
M_{2, d}^{\left(k_{1}, k_{2}\right),\left(e_{1}, e_{2}\right),\left(h_{1}, h_{2}\right)}= & \delta_{k_{1}, 2 k_{2}} O\left(k_{1} q^{-k_{2} / 2}\right)+O\left(k_{1} q^{-k_{2} / 2-k_{1} / 6}+q^{-(1 / 2-1 / p)\left(k_{1}+k_{2}\right)}\right) \\
& + \begin{cases}\delta_{k_{1}, k_{2}} k_{1}\left(1+O\left(q^{-k_{1} / 2}\right)\right), & \left(e_{1}, e_{2}\right)=(1,-1), h_{1}=h_{2}, \\
0, & \text { otherwise }\end{cases}
\end{aligned}
$$

where

$$
\delta_{k_{1}, k_{2}}= \begin{cases}1, & k_{1}=k_{2} \\ 0, & k_{1} \neq k_{2}\end{cases}
$$

Before we proceed with the proof, we would like to make a few remarks. In the instances when we apply this result, we will choose $K=c d$, for $0<c<1 / 2$, and therefore $k_{1}, k_{2} \leq K$ will imply that $k_{1}+k_{2}<d$, and will be able to apply Theorem 7.2 for all values of $k_{1}, k_{2}$ under consideration. Also note that the condition $k_{1} \geq$ $k_{2}>0$ does not restrict the validity of the statement, since $M_{2, d}^{\left(k_{2}, k_{1}\right),(1,-1),\left(h_{1}, h_{2}\right)}=$

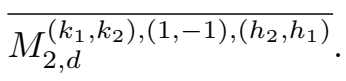


Proof. From Lemma 7.1,

$$
M_{2, d}^{\left(k_{1}, k_{2}\right),\left(e_{1}, e_{2}\right),\left(h_{1}, h_{2}\right)}=q^{-\left(k_{1}+k_{2}\right) / 2}\left(e_{p, e_{1} h_{1} k_{1}+e_{2} h_{2} k_{2}} \sum_{\substack{m\left|\left(k_{1}, k_{2}\right) \\ m p \nmid k_{1}, k_{2} \\ m p\right|\left(e_{1} h_{1} k_{1}+e_{2} h_{2} k_{2}\right)}} \pi(m) m^{2}+e_{p, k_{1}} e_{p, k_{2}} q^{\left(k_{1}+k_{2}\right) / p}\right),
$$

where $\pi(m)$ denotes the number of monic irreducible polynomials of degree $m$ over $\mathbb{F}_{q}[X]$. The prime number theorem for function fields (see [15], Theorem 2.2) states that $\pi(m)=\frac{q^{m}}{m}+O\left(\frac{q^{m / 2}}{m}\right)$.

When $k_{1}=k_{2}$, the conditions on the summation indices become $m \mid k_{1}, m p \nmid k_{1}$, and $m p \mid\left(e_{1} h_{1}+e_{2} h_{2}\right) k_{1}$, a contradiction unless $p \mid\left(e_{1} h_{1}+e_{2} h_{2}\right)$. Owing to the range in which the $h_{1}, h_{2}$ take values, this can only happen when $e_{1}=-e_{2}$ and $h_{1}=h_{2}$. In this case, one obtains

$$
\sum_{\substack{m \mid k_{1} \\ m p \nmid k_{1}}} \pi(m) m^{2}=k_{1} q^{k_{1}}+O\left(k_{1} q^{k_{1} / 2}\right) .
$$

On the other hand, when $k_{1}=2 k_{2}$, one gets

$$
\sum_{\substack{m\left|k_{2} \\ m p \nmid k_{2} \\ m p\right|\left(2 e_{1} h_{1}+e_{2} h_{2}\right) k_{2}}} \pi(m) m^{2}=O\left(k_{2} q^{k_{2}}\right)=O\left(k_{1} q^{k_{1} / 2}\right) .
$$

Finally, if $k_{1}>k_{2}$ but $k_{1} \neq 2 k_{2}$, we have $\left(k_{1}, k_{2}\right) \leq k_{1} / 3$ and

$$
\sum_{\substack{m \mid\left(k_{1}, k_{2}\right) \\ m p \nmid k_{1}, k_{2} \\\left(e_{1} h_{1} k_{1}+e_{2} h_{2} k_{2}\right)}} \pi(m) m^{2}=O\left(k_{1} q^{k_{1} / 3}\right) .
$$

This concludes the proof of the theorem.

Finally, we are able to compute the covariances.

Theorem 7.3. Let $h_{1}, h_{2}$ be integers such that $0<h_{1}, h_{2} \leq(p-1) / 2$. Then for any $K$ with $\max \{1,1 /|\mathcal{I}|\}<K<d / 2$,

$$
\begin{aligned}
\left\langle S^{ \pm}\left(K, f, \psi^{h_{1}}\right) S^{ \pm}\left(K, f, \psi^{h_{2}}\right)\right\rangle & =\left\langle S^{ \pm}\left(K, f, \psi^{h_{1}}\right) S^{\mp}\left(K, f, \psi^{h_{2}}\right)\right\rangle \\
& = \begin{cases}\frac{1}{\pi^{2}} \log (K|\mathcal{I}|)+O(1), & h_{1}=h_{2} \\
O(1), & h_{1} \neq h_{2} .\end{cases}
\end{aligned}
$$

Proof. By definition,

$$
\begin{aligned}
& \left\langle S^{ \pm}\left(K, f, \psi^{h_{1}}\right) S^{ \pm}\left(K, f, \psi^{h_{2}}\right)\right\rangle \\
& =\sum_{k_{1}, k_{2}=1}^{K} \widehat{I}_{K}^{ \pm}\left(k_{1}\right) \widehat{I}_{K}^{ \pm}\left(k_{2}\right) M_{2, d}^{\left(k_{1}, k_{2}\right),(1,1),\left(h_{1}, h_{2}\right)}+\widehat{I}_{K}^{ \pm}\left(k_{1}\right) \widehat{I}_{K}^{ \pm}\left(-k_{2}\right) M_{2, d}^{\left(k_{1}, k_{2}\right),(1,-1),\left(h_{1}, h_{2}\right)} \\
& \quad+\widehat{I}_{K}^{ \pm}\left(-k_{1}\right) \widehat{I}_{K}^{ \pm}\left(k_{2}\right) M_{2, d}^{\left(k_{1}, k_{2}\right),(-1,1),\left(h_{1}, h_{2}\right)}+\widehat{I}_{K}^{ \pm}\left(-k_{1}\right) \widehat{I}_{K}^{ \pm}\left(-k_{2}\right) M_{2, d}^{\left(k_{1}, k_{2}\right),(-1,-1),\left(h_{1}, h_{2}\right)} .
\end{aligned}
$$


Then, by repeated use of Theorem 7.2 and Proposition 5.2, the summation over $k_{1}, k_{2}$ is $O(1)$ if $h_{1} \neq h_{2}$. If $h_{1}=h_{2}$ then

$$
\begin{aligned}
\left\langle S^{ \pm}\left(K, f, \psi^{h_{1}}\right)^{2}\right\rangle & =2 \sum_{k_{1}=1}^{K} \widehat{I}_{K}^{ \pm}\left(k_{1}\right) \widehat{I}_{K}^{ \pm}\left(-k_{1}\right) k_{1}+O(1)=2 \sum_{k_{1} \geq 1} \widehat{I}_{K}^{ \pm}\left(k_{1}\right)^{2} k_{1}+O(1) \\
& =\frac{1}{\pi^{2}} \log (K|\mathcal{I}|)+O(1)
\end{aligned}
$$

by applying Proposition 5.1. The proof for $\left\langle S^{ \pm}\left(K, f, \psi^{h_{1}}\right) S^{\mp}\left(K, f, \psi^{h_{2}}\right)\right\rangle$ follows along exactly the same lines.

Corollary 7.4. For any $K$ with $\max \{1,1 /|\mathcal{I}|\}<K<d / 2$,

$$
\left\langle S^{ \pm}\left(K, C_{f}\right)^{2}\right\rangle=\left\langle S^{+}\left(K, C_{f}\right) S^{-}\left(K, C_{f}\right)\right\rangle=\frac{2(p-1)}{\pi^{2}} \log (K|\mathcal{I}|)+O(1) .
$$

Proof. First, we note that

$$
\left\langle S^{ \pm}\left(K, C_{f}\right)^{2}\right\rangle=\sum_{h_{1}, h_{2}=1}^{p-1}\left\langle S^{ \pm}\left(K, f, \psi^{h_{1}}\right) S^{ \pm}\left(K, f, \psi^{h_{2}}\right)\right\rangle .
$$

Note that by Theorem 7.3, the mixed average contributes $\frac{1}{\pi^{2}} \log (K|\mathcal{I}|)+O(1)$ for each term where $h_{1}=h_{2}$ or $h_{1}=p-h_{2}$. The proof for $\left\langle S^{+}\left(K, C_{f}\right) S^{-}\left(K, C_{f}\right)\right\rangle$ is identical.

\section{Third moment}

Let $k_{1}, k_{2}, k_{3}$ be positive integers, $e_{1}, e_{2}, e_{3}$ take values \pm 1 , and $h_{1}, h_{2}, h_{3}$ be integers such that $p \nmid h_{i}$. Denote $\mathbf{k}=\left(k_{1}, k_{2}, k_{3}\right), \mathbf{e}=\left(e_{1}, e_{2}, e_{3}\right)$, and $\mathbf{h}=\left(h_{1}, h_{2}, h_{3}\right)$. For every $\boldsymbol{\alpha}=\left(\alpha_{1}, \alpha_{2}, \alpha_{3}\right) \in \mathbb{F}_{q^{k_{1}}} \times \mathbb{F}_{q^{k_{2}}} \times \mathbb{F}_{q^{k_{3}}}$, set

$$
m_{3, d}^{\mathbf{k}, \mathbf{e}, \mathbf{h}}(\boldsymbol{\alpha})=\left\langle\psi\left(e_{1} h_{1} \operatorname{tr}_{k_{1}} f\left(\alpha_{1}\right)+e_{2} h_{2} \operatorname{tr}_{k_{2}} f\left(\alpha_{2}\right)+e_{3} h_{3} \operatorname{tr}_{k_{3}} f\left(\alpha_{3}\right)\right)\right\rangle,
$$

and

$$
M_{3, d}^{\mathbf{k}, \mathbf{e}, \mathbf{h}}=\sum_{\substack{\alpha_{i} \in \mathbb{F} \\ i=1,2,3}} q^{-\left(k_{1}+k_{2}+k_{3}\right) / 2} m_{3, d}^{\mathbf{k}, \mathbf{e}, \mathbf{h}}(\boldsymbol{\alpha}) .
$$

In an analogous manner to Section 7, one can prove the following.

Lemma 8.1. Let $p \nmid h_{1} h_{2} h_{3}$ and let $e_{1}, e_{2}, e_{3} \in\{-1,1\}$. Assume $k_{1}, k_{2}, k_{3}>0$ and $k_{1}+k_{2}+k_{3}<d$. For $i=1,2,3 \alpha_{i}$ be an element of $\mathbb{F}_{q^{k_{i}}}$ with minimal polynomial $g_{i}$ over $\mathbb{F}_{q}$ of degree $u_{i}$. We have $m_{3, d}^{\mathbf{k}, \mathbf{e}, \mathbf{h}}(\boldsymbol{\alpha})=1$ in any of the following cases:

- $g_{1}=g_{2}=g_{3}, p \mid \frac{\left(e_{1} h_{1} k_{1}+e_{2} h_{2} k_{2}+e_{3} h_{3} k_{3}\right)}{u_{1}}, p \nmid \frac{k_{1} k_{2} k_{3}}{u_{1} u_{2} u_{3}}$.

- $g_{j_{1}}=g_{j_{2}}, p\left|\frac{\left(e_{j_{1}} h_{j_{1}} k_{j_{1}}+e_{j_{2}} h_{j_{2}} k_{j_{2}}\right)}{u_{j_{1}}}, p \nmid \frac{k_{j_{1}} k_{j_{2}}}{u_{j_{1}} u_{j_{2}}}, p\right| \frac{k_{j_{3}}}{u_{j_{3}}}$, where $\left(j_{1}, j_{2}, j_{3}\right)$ is any permutation of $(1,2,3)$.

- $p \mid \frac{k_{i}}{u_{i}}, i=1,2,3$.

Otherwise $m_{3, d}^{\mathbf{k}, \mathbf{e}, \mathbf{h}}(\boldsymbol{\alpha})=0$. 
Theorem 8.2. Assume $k_{1} \geq k_{2} \geq k_{3}>0$ and $k_{1}+k_{2}+k_{3}<d$. Then

$$
\begin{aligned}
M_{3, d}^{\mathbf{k}, \mathbf{e}, \mathbf{h}} & \\
= & M_{1, d}^{k_{1}, e_{1}, h_{1}} M_{2, d}^{\left(k_{2}, k_{3}\right),\left(e_{2}, e_{3}\right),\left(h_{1}, h_{2}\right)}+M_{1, d}^{k_{2}, e_{2}, h_{3}} M_{2, d}^{\left(k_{1}, k_{3}\right),\left(e_{1}, e_{3}\right),\left(h_{1}, h_{3}\right)} \\
& +M_{1, d}^{k_{3}, e_{3}, h_{3}} M_{2, d}^{\left(k_{1}, k_{2}\right),\left(e_{1}, e_{2}\right),\left(h_{1}, h_{2}\right)}-2 M_{1, d}^{k_{1}, e_{1}, h_{1}} M_{1, d}^{k_{2}, e_{2}, h_{2}} M_{1, d}^{k_{3}, e_{3}, h_{3}} \\
& +O\left(\delta_{k_{1}, k_{2}, k_{3}} k_{1}^{2} q^{-k_{1} / 2}+\delta_{k_{1}, k_{2}, 2 k_{3}} k_{1}^{2} q^{-3 k_{1} / 4}\right. \\
& \left.+\delta_{k_{1}, 2 k_{2}, 2 k_{3}} k_{1}^{2} q^{-k_{1} / 2}+k_{1}^{2} q^{-k_{1} / 6-k_{2}-k_{3}}\right) \\
= & e_{p, k_{1}} q^{-(1 / 2-1 / p) k_{1}} M_{2, d}^{\left(k_{2}, k_{3}\right),\left(e_{2}, e_{3}\right),\left(h_{2}, h_{3}\right)}+e_{p, k_{2}} q^{-(1 / 2-1 / p) k_{2}} M_{2, d}^{\left(k_{1}, k_{3}\right),\left(e_{1}, e_{3}\right),\left(h_{1}, h_{3}\right)} \\
& +e_{p, k_{3}} q^{-(1 / 2-1 / p) k_{3}} M_{2, d}^{\left(k_{1}, k_{2}\right),\left(e_{1}, e_{2}\right),\left(h_{1}, h_{2}\right)} \\
& +O\left(\delta_{k_{1}, k_{2}, k_{3}} k_{1}^{2} q^{-k_{1} / 2}+\delta_{k_{1}, k_{2}, 2 k_{3}} k_{1}^{2} q^{-3 k_{1} / 4}\right) \\
& +O\left(\delta_{k_{1}, 2 k_{2}, 2 k_{3}} k_{1}^{2} q^{-k_{1} / 2}+k_{1}^{2} q^{-k_{1} / 6-k_{2}-k_{3}}+q^{-(1 / 2-1 / p)\left(k_{1}+k_{2}+k_{3}\right)}\right) .
\end{aligned}
$$

Proof. We can use induction in the same way as we used it in the proof of Lemma 8.1. The only new term to be considered is given by the case $g_{1}=g_{2}=g_{3}$ and $p u_{1}$ | $\left(e_{1} h_{1} k_{1}+e_{2} h_{2} k_{2}+e_{3} h_{3} k_{3}\right)$. This term yields

$$
q^{-\left(k_{1}+k_{2}+k_{3}\right) / 2} e_{p, e_{1} h_{1} k_{1}+e_{2} h_{2} k_{2}+e_{3} h_{3} k_{3}} \sum_{\substack{m\left|\left(k_{1}, k_{2}, k_{3}\right) \\ m p \nmid k_{1}, k_{2}, k_{3} \\ m p\right|\left(e_{1} h_{1} k_{1}+e_{2} h_{2} k_{2}+e_{3} h_{3} k_{3}\right)}} \pi(m) m^{3} .
$$

Suppose that $k_{1} \geq k_{2} \geq k_{3}$. If $k_{1}=k_{3}$, we have

$$
\sum_{\substack{m\left|k_{1} \\ m p \nmid k_{1} \\ m p\right|\left(e_{1} h_{1}+e_{2} h_{2}+e_{3} h_{3}\right) k_{1}}} \pi(m) m^{3}=O\left(k_{1}^{2} q^{k_{1}}\right) .
$$

If $k_{1}=2 k_{3}, k_{2}=k_{1}$ or $k_{2}=k_{3}$, we have

$$
\sum_{\substack{m\left|\left(k_{1}, k_{2}, k_{3}\right) \\ m p \nmid k_{3} \\ m p\right|\left(e_{1} h_{1} k_{1}+e_{2} h_{2} k_{2}+e_{3} h_{3} k_{3}\right)}} \pi(m) m^{3}=O\left(k_{1}^{2} q^{k_{1} / 2}\right) .
$$

Finally, for the other cases,

$$
\sum_{\substack{\left.m \mid\left(k_{1}, k_{2}, k_{3}\right) \\ m p \nmid k_{1}, k_{2}, k_{3} \\ h_{1} k_{1}+e_{2} h_{2} k_{2}+e_{3} h_{3} k_{3}\right)}} \pi(m) m^{3}=O\left(k_{1}^{2} q^{k_{1} / 3}\right) .
$$


Theorem 8.3. Let $0<h_{1}, h_{2}, h_{3} \leq(p-1) / 2$. For any $K$ with $\max \{1,1 /|\mathcal{I}|\}<K$ $<d / 3$,

$$
\begin{aligned}
\left\langle S^{ \pm}\right. & \left.\left(K, f, \psi^{h_{1}}\right) S^{ \pm}\left(K, f, \psi^{h_{2}}\right) S^{ \pm}\left(K, f, \psi^{h_{3}}\right)\right\rangle \\
= & \begin{cases}\frac{3 C}{\pi^{2}} \log (K|\mathcal{I}|)+O(1), & h_{1}=h_{2}=h_{3}, \\
\frac{C}{\pi^{2}} \log (K|\mathcal{I}|)+O(1), & h_{j_{1}}=h_{j_{2}} \neq h_{j_{3}},\left(j_{1}, j_{2}, j_{3}\right) \text { a permutation of }(1,2,3), \\
O(1), & h_{i} \text { distinct, }\end{cases}
\end{aligned}
$$

where $C$ is the constant defined in Remark 6.5.

Corollary 8.4. For any $K$ with $\max \{1,1 /|\mathcal{I}|\}<K<d / 3$,

$$
\left\langle S^{ \pm}\left(K, C_{f}\right)^{3}\right\rangle=\frac{6 C(p-1)^{2}}{\pi^{2}} \log (K|\mathcal{I}|)+O(1) .
$$

\section{General moments}

Let $n, k_{1}, \ldots, k_{n}$ be positive integers, let $e_{1}, \ldots, e_{n}$ take values \pm 1 and let $h_{1}, \ldots, h_{n}$ be integers such that $p \nmid h_{i}, 1 \leq i \leq n$. Let $\mathbf{k}=\left(k_{1}, \ldots, k_{n}\right), \mathbf{e}=\left(e_{1}, \ldots, e_{n}\right)$ and $\mathbf{h}=\left(h_{1}, \ldots, h_{n}\right)$. Let $\alpha_{i} \in \mathbb{F}_{q^{k_{i}}}, 1 \leq i \leq n$, and let $\boldsymbol{\alpha}=\left(\alpha_{1}, \ldots, \alpha_{n}\right)$. We define

$$
m_{n, d}^{\mathbf{k}, \mathbf{e}, \mathbf{h}}(\boldsymbol{\alpha})=\left\langle\psi\left(e_{1} h_{1} \operatorname{tr}_{k_{1}} f\left(\alpha_{1}\right)+\cdots+e_{n} h_{n} \operatorname{tr}_{k_{n}} f\left(\alpha_{n}\right)\right)\right\rangle
$$

and

$$
M_{n, d}^{\mathbf{k}, \mathbf{e}, \mathbf{h}}=\sum_{\substack{\alpha_{i} \in \mathbb{F} \\ i=1, \ldots, q_{i}}} q^{-\left(k_{1}+\cdots+k_{n}\right) / 2} m_{n, d}^{\mathbf{k}, \mathbf{e}, \mathbf{h}}(\boldsymbol{\alpha}) .
$$

We are computing in this section the general moments

$$
\left\langle S^{ \pm}(K, f, \psi)^{n}\right\rangle=\sum_{k_{1}, \ldots, k_{n}=1}^{K} \sum_{e_{1}, \ldots, e_{n}= \pm 1} I_{K}^{ \pm}\left(e_{1} k_{1}\right) \cdots I_{K}^{ \pm}\left(e_{n} k_{n}\right) M_{n, d}^{\mathbf{k}, \mathbf{e}}
$$

and

$$
\left\langle S^{ \pm}\left(K, f, \psi^{h_{1}}\right) \cdots S^{ \pm}\left(K, f, \psi^{h_{n}}\right)\right\rangle=\sum_{k_{1}, \ldots, k_{n}=1}^{K} \sum_{\substack{e_{j}= \pm 1, 1 \leq j \leq n}} I_{K}^{ \pm}\left(e_{1} k_{1}\right) \cdots I_{K}^{ \pm}\left(e_{n} k_{n}\right) M_{n, d}^{\mathbf{k}, \mathbf{e}, \mathbf{h}} .
$$

Lemma 9.1. Assume $k_{1}, \ldots, k_{n}>0, k_{1}+\cdots+k_{n}<d$. Let $g_{1}, \ldots, g_{s}$ of degree $u_{1}, \ldots, u_{s}$ respectively be all the distinct minimal polynomials over $\mathbb{F}_{q}$ of $\alpha_{1}, \ldots, \alpha_{n}$ (we allow the possibility that some $\alpha_{i}$ 's are conjugate to each other, thus $s \leq n$ ), and let

$$
\epsilon_{i}=\frac{1}{u_{i}} \sum_{\alpha_{j} \in R\left(g_{i}\right)} k_{j} e_{j} h_{j}, \quad 1 \leq i \leq s
$$

where $R(g)$ is the set of roots of $g$. Then

$$
m_{n, d}^{\mathbf{k}, \mathbf{e}, \mathbf{h}}(\boldsymbol{\alpha})= \begin{cases}1, & \text { if } p \mid \epsilon_{i} \text { for } 1 \leq i \leq s \\ 0, & \text { otherwise }\end{cases}
$$


Proof. As before, we can take the average over the family $\mathcal{F}_{d}$ of polynomials of degree $d$ without the condition that $a_{k p}=0$ for $1 \leq k \leq d / p$. Renumbering, suppose that $\alpha_{i}$ has minimal polynomial $g_{i}$ for $1 \leq i \leq s$.

Since $\sum_{i=1}^{s} u_{i} \leq \sum_{i=1}^{s} k_{i}<d$, the map

$$
\tau: \mathcal{F}_{d} \rightarrow \mathbb{F}_{q}[X] /\left(g_{1} \cdots g_{s}\right) \simeq \mathbb{F}_{q^{u_{1}}} \times \cdots \times \mathbb{F}_{q^{u_{s}}}
$$

is exactly $(q-1) q^{d-\left(u_{1}+\cdots+u_{s}\right)}$-to-one, and as $f$ ranges over $\mathcal{F}_{d},\left(f\left(\alpha_{1}\right), \ldots, f\left(\alpha_{s}\right)\right)$ takes every value in $\mathbb{F}_{q^{u_{1}}} \times \cdots \times \mathbb{F}_{q^{u_{s}}}$ the same number of times. Now, the product $\left(\operatorname{tr}_{u_{1}} f\left(\alpha_{1}\right), \ldots, \operatorname{tr}_{u_{s}} f\left(\alpha_{s}\right)\right)$ also takes every value in $\left(\mathbb{F}_{p}\right)^{s}$ the same number of times as $f$ ranges over $\mathcal{F}_{d}$, and the same holds for any linear combination

$$
\gamma_{1} \operatorname{tr}_{u_{1}} f\left(\alpha_{1}\right)+\cdots+\gamma_{s} \operatorname{tr}_{u_{s}} f\left(\alpha_{s}\right),
$$

unless $p$ divides every $\gamma_{i}$. This shows that each $p$ th root of unity occurs as many times as

$$
\psi\left(\gamma_{1} \operatorname{tr}_{u_{1}} f\left(\alpha_{1}\right)+\cdots+\gamma_{s} \operatorname{tr}_{u_{s}} f\left(\alpha_{s}\right)\right)
$$

when $p$ does not divide all the $\gamma_{i}$. We now determine the coefficients $\gamma_{i}$ for

$$
m_{n, d}^{\mathbf{k}, \mathbf{e}, \mathbf{h}}(\boldsymbol{\alpha})=\sum_{f \in \mathcal{F}_{d}} \psi\left(e_{1} h_{1} \operatorname{tr}_{k_{1}} f\left(\alpha_{1}\right)+\cdots+e_{n} h_{n} \operatorname{tr}_{k_{n}} f\left(\alpha_{n}\right)\right) .
$$

Recall that $\operatorname{tr}_{k_{i}} f\left(\alpha_{i}\right)=\frac{k_{i}}{u_{i}} \operatorname{tr}_{u_{i}} f\left(\alpha_{i}\right)$ for $i=1, \ldots, s$. Let

$$
\epsilon_{i}=\frac{1}{u_{i}} \sum_{\alpha_{j} \in R\left(g_{i}\right)} e_{j} h_{j} k_{j}, \quad 1 \leq i \leq s .
$$

Then $\gamma_{i}=\epsilon_{i}$, i.e.,

$$
m_{n, d}^{\mathbf{k}, \mathbf{e}, \mathbf{h}}(\boldsymbol{\alpha})=\sum_{f \in \mathcal{F}_{d}} \psi\left(\epsilon_{1} \operatorname{tr}_{u_{1}} f\left(\alpha_{1}\right)+\cdots+\epsilon_{s} \operatorname{tr}_{u_{s}} f\left(\alpha_{s}\right)\right),
$$

which implies that $m_{n, d}^{\mathbf{k}, \mathbf{e}, \mathbf{h}}(\boldsymbol{\alpha})$ takes the value 1 if $p \mid \epsilon_{i}$ for $1 \leq i \leq s$, and 0 otherwise.

Recall that $\pi(m)$ denotes the number of monic irreducible polynomials of degree $m$ in $\mathbb{F}_{q}[X]$.

Lemma 9.2. Assume $k_{1}, \ldots, k_{n}>0, k_{1}+\cdots+k_{n}<d$. Then $M_{n, d}^{\mathbf{k}, \mathbf{e}, \mathbf{h}}$ is bounded by a sum of terms made of products of elementary terms of the type

$$
q^{-\left(j_{1}+\cdots+j_{r}\right) / 2} \sum_{\substack{m\left|\left(j_{1}, \ldots, j_{r}\right) \\ m p\right| \sum_{i=1}^{r} e_{i} h_{i} j_{i}}} \pi(m) m^{r}
$$

where the indices $j_{1}, \ldots, j_{r}$ of the elementary terms appearing in each product are in bijection with $k_{1}, \ldots, k_{n}$.

Let $N_{n, d}^{\mathbf{k}, \mathbf{e}, \mathbf{h}}$ be the sum of the terms made exclusively of products of elementary terms

$$
q^{-\left(j_{1}+j_{2}\right) / 2} \sum_{\substack{m\left|\left(j_{1}, j_{2}\right) \\ m p\right| e_{1} h_{1} j_{1}+e_{2} h_{2} j_{2}}} \pi(m) m^{2} .
$$


If $n$ is odd, these terms will also be multiplied by an elementary term

$$
e_{p, j} q^{-j / 2} \sum_{\substack{m|j \\ m p| e j}} \pi(m) m=e_{p, j} \sum_{m \mid \frac{j}{p}} \pi(m) m=e_{p, j} \# \mathbb{F}_{q^{j / p}}=e_{p, j} q^{j / p} .
$$

Let $E_{n, d}^{\mathbf{k}, \mathbf{e}, \mathbf{h}}$ be the sum of all the other terms appearing in $M_{n, d}^{\mathbf{k}, \mathbf{e}, \mathbf{h}}$. Then, $M_{n, d}^{\mathbf{k}, \mathbf{e}, \mathbf{h}}=$ $N_{n, d}^{\mathbf{k}, \mathbf{e}, \mathbf{h}}+O\left(E_{n, d}^{\mathbf{k}, \mathbf{e}, \mathbf{h}}\right)$.

Proof. We first remark that the number of $\left(\alpha_{1}, \ldots, \alpha_{t}\right) \in \mathbb{F}_{q^{k_{1}}} \times \cdots \times \mathbb{F}_{q^{k_{t}}}$ which are conjugate over $\mathbb{F}_{q}$ is

$$
\sum_{m \mid\left(k_{1}, \ldots, k_{t}\right)} \pi(m) m^{t}
$$

Using Lemma 9.1, we then have to count the contribution coming from the $\boldsymbol{\alpha}=\left(\alpha_{1}, \ldots, \alpha_{n}\right)$ such that $p \mid \epsilon_{i}$ for $1 \leq i \leq s$. Let $\mathcal{P}$ be the set of partitions of $n$ in $s$ subsets $T_{1}, \ldots, T_{s}$. Let $k\left(T_{j}\right)$ be the gcd of the $k_{i}$ such that $i \in T_{j}$ and let $s\left(T_{j}\right)=\sum_{i \in T_{j}} e_{i} h_{i} k_{i}$. Then, for any such partition, the number of $\alpha=\left(\alpha_{1}, \ldots, \alpha_{n}\right) \in$ $\mathbb{F}_{q^{k_{1}}} \times \cdots \times \mathbb{F}_{q^{k_{n}}}$ such that $\alpha_{i}$ is a root of $g_{j}$ when $i \in T_{j}$ is less than or equal to

$$
\sum_{\substack{m\left|k\left(T_{1}\right) \\ m p\right| s\left(T_{1}\right)}} \pi(m) m^{\left|T_{1}\right|} \cdots \sum_{\substack{m\left|k\left(T_{s}\right) \\ m p\right| s\left(T_{s}\right)}} \pi(m) m^{\left|T_{s}\right|} .
$$

This proves the first statement of the lemma. We remark that the above count is an overcount, as it may also count polynomials $g_{1}, \ldots, g_{s}$ which are not distinct. For example, the number of $\left(\alpha_{1}, \alpha_{2}, \alpha_{3}, \alpha_{4}\right) \in \mathbb{F}_{q^{j_{1}}} \times \cdots \times \mathbb{F}_{q^{j_{4}}}$ with minimal polynomials $g_{1}=g_{2}, g_{3}=g_{4}$ and $g_{1} \neq g_{3}$ is

$$
\begin{gathered}
q^{-\left(j_{1}+\cdots+j_{4}\right) / 2} \sum_{\substack{m\left|\left(j_{1}, j_{2}\right) \\
m p\right| e_{1} h_{1} j_{1}+e_{2} h_{2} j_{2}}} \pi(m) m^{2} \sum_{\substack{m\left|\left(j_{3}, j_{4}\right) \\
m p\right| e_{3} h_{3} j_{3}+e_{4} h_{4} j_{4}}} \pi(m) m^{2} \\
-q^{-\left(j_{1}+\cdots+j_{4}\right) / 2} \sum_{\substack{m\left|\left(j_{1}, \ldots, j_{4}\right) \\
m p\right| e_{1} h_{1} j_{1}+\cdots+e_{4} h_{4} j_{4}}} \pi(m) m^{4},
\end{gathered}
$$

which can be written as a term in $N_{n, d}^{\mathbf{k}, \mathbf{e}, \mathbf{h}}$ and a term in $E_{n, d}^{\mathbf{k}, \mathbf{e}, \mathbf{h}}$. The general case is similar. Suppose that $n=2 \ell$ is even. Then, using inclusion-exclusion, the number of $\left(\alpha_{1}, \ldots, \alpha_{n}\right) \in\left(\mathbb{F}_{q^{k_{1}}}, \ldots, \mathbb{F}_{q^{k_{n}}}\right)$ such that $\alpha_{i}$ and $\alpha_{\ell+i}$ have minimal polynomial $g_{i}$, and all the $g_{i}$ are distinct can be written as

$$
\begin{aligned}
& q^{-\left(k_{1}+\cdots+k_{2 \ell}\right) / 2}\left(\sum_{\substack{m\left|\left(k_{1}, k_{\ell+1}\right) \\
m p\right| e_{1} h_{1} k_{1}+e_{\ell+1} h_{\ell+1} k_{\ell+1}}} \pi(m) m^{2} \cdots \sum_{\substack{m\left|\left(k_{\ell}, k_{2 \ell}\right) \\
m p\right| e_{\ell} h_{\ell} k_{\ell}+e_{2 \ell} h_{2 \ell} k_{e \ell}}} \pi(m) m^{2}\right) \\
& +S\left(k_{1}, \ldots, k_{n}\right)
\end{aligned}
$$

where $S\left(k_{1}, \ldots, k_{n}\right)$ is a sum of terms in $E_{n, d}^{\mathbf{k}, \mathbf{e}, \mathbf{h}}$.

The case of $n=2 \ell+1$ follows similarly, taking into account that one has to multiply by the factor $e_{p, k_{n}} q^{-k_{n} / 2} \sum_{\substack{m\left|k_{n} \\ m p\right| e k_{n}}} \pi(m) m$. 
We now compute

$$
\left\langle S^{ \pm}\left(K, f, \psi^{h_{1}}\right) \cdots S^{ \pm}\left(K, f, \psi^{h_{n}}\right)\right\rangle=\sum_{\substack{k_{1}, \ldots, k_{n}=1 \\ e_{1}, \ldots, e_{n}= \pm 1}}^{K} I_{K}^{ \pm}\left(e_{1} k_{1}\right) \cdots I_{K}^{ \pm}\left(e_{n} k_{n}\right) M_{n, d}^{\mathbf{k}, \mathbf{e}, \mathbf{h}} .
$$

We will use $K=c d$ where $0<c<1 / n$. Then, $k_{i} \leq K$ implies that $k_{1}+\cdots+k_{n}<d$, and we can apply the lemmas above.

Using Lemma 9.2, we have to compute sums of the type

$$
\sum_{k=1}^{K} \widehat{I}_{K}^{ \pm}(k) q^{-(1 / 2-1 / p) k}=C(K)=O(1)
$$

and for $r \geq 2$

$$
\sum_{k_{1}, \ldots, k_{r}=1}^{K} \widehat{I}_{K}^{ \pm}\left(e_{1} k_{1}\right) \cdots \widehat{I}_{K}^{ \pm}\left(e_{r} k_{r}\right) q^{-\left(k_{1}+\cdots+k_{r}\right) / 2} \sum_{\substack{m\left|\left(k_{1}, \ldots, k_{r}\right) \\ m p\right| \sum_{i=1}^{f} e_{i} h_{i} k_{i}}} \pi(m) m^{r} .
$$

If $r=2$, we have when $p \mid e_{1} h_{1} k_{1}+e_{2} h_{2} k_{2}$

$$
\begin{aligned}
& \sum_{k_{1}, k_{2}=1}^{K} \widehat{I}_{K}^{ \pm}\left(e_{1} k_{1}\right) \widehat{I}_{K}^{ \pm}\left(e_{2} k_{2}\right) q^{-\left(k_{1}+k_{2}\right) / 2} \sum_{\substack{m\left|\left(k_{1}, k_{2}\right) \\
m p\right|\left(e_{1} h_{1} k_{1}+e_{2} h_{2} k_{2}\right)}} \pi(m) m^{2} \\
& = \begin{cases}\frac{1}{2 \pi^{2}} \log (K|\mathcal{I}|)+O(1), & e_{1} h_{1}+e_{2} h_{2} \equiv 0 \bmod p, \\
O(1), & \text { otherwise }\end{cases}
\end{aligned}
$$

as we computed in the proof of Theorems 7.2 and 7.3. (In those theorems we had the extra condition $m p \nmid k_{1}, k_{2}$ in the sum, but those additional terms only add an $O(1)$ to the final sum, and we can ignore them.)

For the other terms, we have

Lemma 9.3. Let $r>2$, then

$$
S:=\sum_{k_{1}, \ldots, k_{r}=1}^{K} \widehat{I}_{K}^{ \pm}\left(k_{1}\right) \cdots \widehat{I}_{K}^{ \pm}\left(k_{r}\right) q^{-\left(k_{1}+\cdots+k_{r}\right) / 2} \sum_{\substack{m \mid\left(k_{1}, \ldots, k_{r}\right) \\ m p \nmid\left(k_{1}, \ldots, k_{r}\right)}} \pi(m) m^{r}=O(1) .
$$

Proof. Suppose for the moment that $k_{1} \geq \cdots \geq k_{r}$. If $k_{1}=k_{r}$, we have

$$
\sum_{\substack{m \mid\left(k_{1}, \ldots, k_{r}\right) \\ m p \nmid\left(k_{1}, \ldots, k_{r}\right)}} \pi(m) m^{f}=O\left(k_{1}^{r} q^{k_{1}}\right) .
$$

If $k_{1}=2 k_{r}$, and all the other $k_{i}$ are equal to $k_{1}$ or $k_{r}$, we have

$$
\sum_{\substack{m \mid\left(k_{1}, \ldots, k_{r}\right) \\ m p \nmid\left(k_{1}, \ldots, k_{r}\right)}} \pi(m) m^{r}=O\left(k_{1}^{r} q^{k_{1} / 2}\right) .
$$

In all the other cases,

$$
\sum_{\substack{m \mid\left(k_{1}, \ldots, k_{r}\right) \\ m p \nmid\left(k_{1}, \ldots, k_{r}\right)}} \pi(m) m^{r}=O\left(k_{1}^{r} q^{k_{1} / 3}\right) .
$$


Putting things together, we obtain

$$
\begin{aligned}
S & \ll \sum_{k=1}^{K} \widehat{I}_{K}^{ \pm}(k)^{r} k^{r} q^{-(r-2) k / 2}+\sum_{\ell=1}^{r-1} \sum_{k=1}^{K} \widehat{I}_{K}^{ \pm}(2 k)^{\ell} \widehat{I}_{K}^{ \pm}(k)^{r-\ell} k^{r} q^{(1-r / 2-\ell / 2) k} \\
& +\sum_{k_{1}, \ldots, k_{r}=1}^{K} \widehat{I}_{K}^{ \pm}\left(k_{1}\right) \cdots \widehat{I}_{K}^{ \pm}\left(k_{r}\right) k_{1}^{r} q^{-k_{1} / 6-\left(k_{2}+\cdots+k_{r}\right) / 2} \\
& \ll 1
\end{aligned}
$$

by Proposition 5.2.

Theorem 9.4. For any $K$ with $\max \{1,1 /|\mathcal{I}|\}<K<d / n$

$$
\left\langle S^{ \pm}(K, f, \psi)^{n}\right\rangle= \begin{cases}\frac{(2 \ell) !}{\ell !\left(2 \pi^{2}\right)^{\ell}} \log ^{\ell}(K|\mathcal{I}|)\left(1+O\left(\log ^{-1}(K|\mathcal{I}|)\right)\right) & n=2 \ell \\ C \frac{(2 \ell+1) !}{\ell !\left(2 \pi^{2}\right)^{\ell}} \log ^{\ell}(K|\mathcal{I}|)\left(1+O\left(\log ^{-1}(K|\mathcal{I}|)\right)\right) & n=2 \ell+1\end{cases}
$$

where $C$ is defined in Remark 6.5.

Proof. By Lemmas 9.2 and 9.3, we observe that the leading term in $S^{ \pm}(K, f, \psi)^{n}$ will come from the contributions $N_{n, d}^{\mathbf{k}, \mathbf{e}}$. By equation (9.2), if $n=2 \ell$, the leading terms are of the form

$$
\left(\frac{1}{2 \pi^{2}} \log (K|\mathcal{I}|)\right)^{\ell}
$$

and if $n=2 \ell+1$, the leading terms are of the form

$$
C\left(\frac{1}{2 \pi^{2}} \log (K|\mathcal{I}|)\right)^{\ell}
$$

The final coefficient is obtained by counting the numbers of ways to choose the $\ell$ (or $\ell+1)$ coefficients $k_{i}^{\prime} s$ with positive sign $\left(e_{i}=1\right)$ and to pair them with those with negative sign $\left(e_{j}=-1\right)$.

As $S^{ \pm}(K, f, \psi)=S^{ \pm}(K, f, \bar{\psi})$, it is sufficient to study the sum of $S^{ \pm}\left(K, f, \psi^{j}\right)$ for $j$ up to $(p-1) / 2$ rather than $p-1$.

We let

$$
\delta_{n}(C)= \begin{cases}1 & n=2 \ell, \\ C & n=2 \ell+1 .\end{cases}
$$

Theorem 9.5. Let $\ell=\left\lfloor\frac{n}{2}\right\rfloor$. Let $0<h_{1}, \ldots, h_{n} \leq(p-1) / 2$. Then for any $K$ with $\max \{1,1 /|\mathcal{I}|\}<K<d / n$,

$$
\begin{aligned}
& \left\langle S^{ \pm}\left(K, f, \psi^{h_{1}}\right) \cdots S^{ \pm}\left(K, f, \psi^{h_{n}}\right)\right\rangle \\
& \quad=\delta_{n}(C) \frac{\Delta\left(h_{1}, \ldots, h_{n}\right)}{\left(2 \pi^{2}\right)^{\ell}} \log ^{\ell}(K|\mathcal{I}|)\left(1+O\left(\log ^{-1}(K|\mathcal{I}|)\right)\right)
\end{aligned}
$$

where $C$ is defined in Remark 6.5 and

$$
\begin{aligned}
& \Delta\left(h_{1}, \ldots, h_{n}\right)=\#\left\{\left(e_{1}, \ldots, e_{n}\right) \in\{-1,1\}, \sigma \in \mathbb{S}_{n} \mid\right. \\
&\left.e_{1} h_{\sigma(1)}+e_{2} h_{\sigma(2)} \equiv \cdots \equiv e_{2 \ell-1} h_{\sigma(2 \ell-1)}+e_{2 \ell} h_{\sigma(2 \ell)} \equiv 0 \bmod p\right\}
\end{aligned}
$$

where $\mathbb{S}_{n}$ denotes the permutations of the set of $n$ elements. 
Proof. By Lemmas 9.2 and 9.3, we observe that the leading term in the product $S^{ \pm}\left(K, f, \psi^{h_{1}}\right) \cdots S^{ \pm}\left(K, f, \psi^{h_{n}}\right)$ will come from the contributions $N_{n, d}^{\mathbf{k}, \mathbf{e}, \mathbf{h}}$. By Theorem 7.3 , if $n=2 \ell$, the leading terms are of the form

$$
\left(\frac{1}{2 \pi^{2}} \log (K|\mathcal{I}|)\right)^{\ell}
$$

and if $n=2 \ell+1$, the leading terms are of the form

$$
C\left(\frac{1}{2 \pi^{2}} \log (K|\mathcal{I}|)\right)^{\ell} .
$$

The final coefficient is obtained by counting the numbers of ways to choose the $\ell$ (or $\ell+1)$ coefficients $k_{i}$ with positive $\operatorname{sign}\left(e_{i}=1\right)$ and to pair them with $k_{j}$ with negative sign $\left(e_{j}=-1\right)$ in such a way that $p$ divides $e_{i} h_{i}+e_{j} h_{j}$.

We note that if $n=2 \ell$,

$$
\sum_{h_{1}, \ldots, h_{n}=1}^{(p-1) / 2} \Delta\left(h_{1}, \ldots, h_{n}\right)=\frac{(p-1)^{\ell}(2 \ell) !}{2^{\ell} \ell !} .
$$

There are $\frac{(2 \ell) !}{\ell ! 2^{\ell}}$ ways of choosing pairs $\left\{e_{i}, e_{j}\right\}$ (because the order does not count inside the pair). For each pair either $e_{i}$ or $e_{j}$ can be negative and the other one positive so there are a total $2^{\ell}$ choices for the signs. Finally, for each pair there are $((p-1) / 2)$ possible values for $h_{i}$ and this determines $h_{j}$.

Remark 9.6. A consequence of Theorem 9.5 is that the moments are given by sums of products of covariances, exactly in the same way as the moments of a multivariate normal distribution. Moreover, the generating function of the moments converges due to (9.3). Therefore, our random variables are jointly normal. Since the variables are uncorrelated (cf. Theorem 7.3), it follows that our random variables are independent.

Recall that

$$
S^{ \pm}\left(K, C_{f}\right)=\sum_{j=1}^{p-1} S^{ \pm}\left(K, f, \psi^{j}\right) .
$$

Theorem 9.7. Assume that $K=d / \log \log (d|\mathcal{I}|), d \rightarrow \infty$ and either $0<|\mathcal{I}|<1$ is fixed or $|\mathcal{I}| \rightarrow 0$ while $d|\mathcal{I}| \rightarrow \infty$. Then

$$
\frac{S^{ \pm}\left(K, C_{f}\right)}{\sqrt{\frac{2(p-1)}{\pi^{2}} \log (d|\mathcal{I}|)}}
$$

has a standard Gaussian limiting distribution when $d \rightarrow \infty$.

Proof. First, we compute the moments and then we normalize them. Let $\ell=\left\lfloor\frac{n}{2}\right\rfloor$. We note that with our choice of $K$, we have

$$
\frac{\log (K|\mathcal{I}|)}{\log (d|\mathcal{I}|)}=1-\frac{\log \log \log (d|\mathcal{I}|)}{\log (d|\mathcal{I}|)}
$$

Therefore, we can replace $\log (K|\mathcal{I}|)$ by $\log (d|\mathcal{I}|)$ in our formulas. 
Recall that $S^{ \pm}\left(K, f, \psi^{j}\right)=S^{ \pm}\left(K, f, \psi^{p-j}\right)$, then

$$
\begin{aligned}
& S^{ \pm}\left(K, C_{f}\right)^{n}=\left(2 \sum_{j=1}^{(p-1) / 2} S^{ \pm}\left(K, f, \psi^{j}\right)\right)^{n} \\
& =2^{n} \sum_{j_{1}, \ldots, j_{n}=1}^{(p-1) / 2} S^{ \pm}\left(K, f, \psi^{j_{1}}\right) \cdots S^{ \pm}\left(K, f, \psi^{j_{n}}\right) .
\end{aligned}
$$

Therefore, we can compute the moment

$$
\left\langle S^{ \pm}\left(K, C_{f}\right)^{n}\right\rangle=2^{n} \sum_{j_{1}, \ldots, j_{n}=1}^{(p-1) / 2}\left\langle S^{ \pm}\left(K, f, \psi^{j_{1}}\right) \cdots S^{ \pm}\left(K, f, \psi^{j_{n}}\right)\right\rangle
$$

and then by Theorem 9.5 this is asymptotic to

$$
\frac{2^{n} \delta_{n}(C)}{\left(2 \pi^{2}\right)^{\ell}} \log ^{\ell}(d|\mathcal{I}|) \sum_{j_{1}, \ldots, j_{n}=1}^{(p-1) / 2} \Delta\left(j_{1}, \ldots, j_{n}\right) .
$$

Finally, we use equation (9.3) to conclude that when $n=2 \ell$,

$$
\left\langle S^{ \pm}\left(K, C_{f}\right)^{n}\right\rangle=\frac{2^{n}(p-1)^{\ell}(2 \ell) !}{2^{\ell} \ell !\left(2 \pi^{2}\right)^{\ell}} \log ^{\ell}(d|\mathcal{I}|)=\frac{(2 \ell) !}{\ell ! \pi^{2 \ell}}(p-1)^{\ell} \log ^{\ell}(d|\mathcal{I}|)
$$

and the variance is asymptotic to $\frac{2(p-1)}{\pi^{2}} \log (d|\mathcal{I}|)$.

Hence, the normalized moment converges to 0 for $n$ odd and for $n$ even,

$$
\lim _{d \rightarrow \infty} \frac{\left\langle S^{ \pm}\left(K, C_{f}\right)^{2 \ell}\right\rangle}{\left(\sqrt{\frac{2(p-1)}{\pi^{2}} \log (d|\mathcal{I}|)}\right)^{2 \ell}}=\frac{(2 \ell) !}{\ell ! 2^{\ell}} .
$$

\section{Proof of main theorem}

We prove in this section that

$$
\frac{N_{\mathcal{I}}\left(C_{f}\right)-2 g|\mathcal{I}|}{\sqrt{\left(2(p-1) / \pi^{2}\right) \log (d|\mathcal{I}|)}}
$$

converges in mean square to

$$
\frac{S^{ \pm}\left(K, C_{f}\right)}{\sqrt{\left(2(p-1) / \pi^{2}\right) \log (d|\mathcal{I}|)}} .
$$

Then, using Theorem 9.7, we obtain the result of Theorem 1.1 since convergence in mean square implies convergence in distribution.

Lemma 10.1. Assume that $K=d / \log \log (d|\mathcal{I}|), d \rightarrow \infty$ and either $0<|\mathcal{I}|<1$ is fixed or $|\mathcal{I}| \rightarrow 0$, while $d|\mathcal{I}| \rightarrow \infty$. Then

$$
\left\langle\left|\frac{N_{\mathcal{I}}\left(C_{f}\right)-(d-1)(p-1)|\mathcal{I}|+S^{ \pm}\left(K, C_{f}\right)}{\sqrt{\left(2(p-1) / \pi^{2}\right) \log (d|\mathcal{I}|)}}\right|^{2}\right\rangle \rightarrow 0
$$


Proof. From equation (6.2) from Section 6, using the Beurling-Selberg polynomials and the explicit formula (Lemma 3.1), we deduce that

$$
\begin{aligned}
\frac{-(p-1)(d-1)}{K+1} & \leq N_{\mathcal{I}}\left(C_{f}\right)-(p-1)(d-1)|\mathcal{I}|+S^{-}\left(K, C_{f}\right) \\
& \leq S^{-}\left(K, C_{f}\right)-S^{+}\left(K, C_{f}\right)+\frac{(p-1)(d-1)}{K+1}
\end{aligned}
$$

and

$$
\begin{aligned}
\frac{-(p-1)(d-1)}{K+1} & \leq-N_{\mathcal{I}}\left(C_{f}\right)+(p-1)(d-1)|\mathcal{I}|-S^{+}\left(K, C_{f}\right) \\
& \leq S^{-}\left(K, C_{f}\right)-S^{+}\left(K, C_{f}\right)+\frac{(p-1)(d-1)}{K+1} .
\end{aligned}
$$

Using these two inequalities to bound the absolute value of the central term, we obtain

$$
\begin{aligned}
& \left\langle\left(N_{\mathcal{I}}\left(C_{f}\right)-(p-1)(d-1)|\mathcal{I}|+S^{ \pm}\left(K, C_{f}\right)\right)^{2}\right\rangle \\
& \leq \max \left\{\left(\frac{(p-1)(d-1)}{K+1}\right)^{2},\left\langle\left(S^{-}\left(K, C_{f}\right)-S^{+}\left(K, C_{f}\right)+\frac{(p-1)(d-1)}{K+1}\right)^{2}\right\rangle\right\} \\
& \leq\left(\frac{(p-1)(d-1)}{K+1}\right)^{2}+\max \left\{0,\left\langle\left(S^{-}\left(K, C_{f}\right)-S^{+}\left(K, C_{f}\right)\right)^{2}\right\rangle\right. \\
& \left.\quad+2 \frac{(p-1)(d-1)}{K+1}\left\langle S^{-}\left(K, C_{f}\right)-S^{+}\left(K, C_{f}\right)\right\rangle\right\} .
\end{aligned}
$$

Now using the estimate in the proof of Theorem 6.1 , we have that

$$
\left\langle S^{-}\left(K, C_{f}\right)-S^{+}\left(K, C_{f}\right)\right\rangle=\left\langle S^{-}\left(K, C_{f}\right)\right\rangle-\left\langle S^{+}\left(K, C_{f}\right)\right\rangle=O(1) .
$$

For the remaining term we note that

$$
\begin{aligned}
& \left\langle\left(S^{-}\left(K, C_{f}\right)-S^{+}\left(K, C_{f}\right)\right)^{2}\right\rangle \\
& \quad=\left\langle\left(S^{-}\left(K, C_{f}\right)\right)^{2}\right\rangle+\left\langle\left(S^{+}\left(K, C_{f}\right)\right)^{2}\right\rangle-2\left\langle\sum_{j_{1}, j_{2}=1}^{p-1} S^{-}\left(K, f, \psi^{j_{1}}\right) S^{+}\left(K, f, \psi^{j_{2}}\right)\right\rangle .
\end{aligned}
$$

By Corollary 7.4, this equals

$$
\frac{4(p-1)}{\pi^{2}} \log (d|\mathcal{I}|)+O(1)-\frac{4(p-1)}{\pi^{2}} \log (d|\mathcal{I}|)+O(1)=O(1) .
$$

Therefore,

$$
\left\langle\left(N_{\mathcal{I}}\left(C_{f}\right)-(p-1)(d-1)|\mathcal{I}|+S^{ \pm}\left(K, C_{f}\right)\right)^{2}\right\rangle=O\left(\left(\frac{(p-1)(d-1)}{K+1}\right)^{2}\right)
$$

and

$$
\left\langle\left(\frac{N_{\mathcal{I}}\left(C_{f}\right)-(p-1)(d-1)|\mathcal{I}|+S^{ \pm}\left(K, C_{f}\right)}{\sqrt{\left(2(p-1) / \pi^{2}\right) \log (d|\mathcal{I}|)}}\right)^{2}\right\rangle \rightarrow 0
$$

when $d$ tends to infinity and $K=d / \log \log (d|\mathcal{I}|)$. 
Remark 10.2. Proposition 1.3 is proved in a similar way. For this, one uses Theorem 9.4 to examine the moments of

$$
\frac{S^{ \pm}(K, f, \psi)+S^{ \pm}(K, f, \bar{\psi})}{\sqrt{\frac{4}{\pi^{2}} \log (d|\mathcal{I}|)}}=\frac{2 S^{ \pm}(K, f, \psi)}{\sqrt{\frac{4}{\pi^{2}} \log (d|\mathcal{I}|)}} .
$$

\section{Acknowledgments}

The authors thank Zeév Rudnick for many useful discussions while preparing this paper. The authors are also grateful to Louis-Pierre Arguin, Andrew Granville and Rachel Pries for helpful conversations related to this work. A. B., B. F. and K. S. acknowledge the Centre de Recherche Mathématique (CRM) and the Mathematical Sciences Research Institute (MSRI) for their hospitality.

This work was supported by the National Science Foundation of USA [grant DMS1201446 to B. F.], the Simons Foundation [no. 244988 to A. B.] the UCSD Hellman Fellows Program [2012-2013 Hellman Fellowship to A. B.], the Natural Sciences and Engineering Research Council of Canada [Discovery Grant 155635-2008 to C. D., 355412-2008 to M. L.] and the Fonds de recherche du Québec - Nature et technologies [grants 144987 to M. L., 166534 to C. D. and M. L.]

\section{References}

[1] A. Bucur, C. David, B. Feigon and M. Lalín, Fluctuations in the number of points on smooth plane curves over finite fields, J. Number Theory 130(11) (2010), 2528-2541.

[2] - Statistics for traces of cyclic trigonal curves over finite fields, Int. Math. Res. Not. IMRN (5) (2010), 932-967.

[3] —, Statistics for traces of cyclic trigonal curves over finite fields, in WIN-Women in Numbers: Research Directions in Number Theory (Fields Institute Communications Series), Vol. 60, Amer. Math. Soc., Providence, RI (2011), pp. 121-143.

[4] A. Bucur and K.S. Kedlaya, The probability that a complete intersection is smooth. J. Théor. Nombres Bordeaux 24(3) (2012), 541-556.

[5] P. Deligne, La conjecture de Weil. I, Inst. Hautes Études Sci. Publ. Math. 43 (1974), 273-307.

[6] - La conjecture de Weil. II, Inst. Hautes Études Sci. Publ. Math. 52 (1980), 137-252.

[7] A. Entin, On the distribution of zeroes of Artin-Schreier L-functions, Geom. Funct. Anal. 22(5) (2012), 1322-1360.

[8] D. Faifman and Z. Rudnick, Statistics of the zeros of zeta functions in families of hyperelliptic curves over a finite field, Compos. Math. 146(1) (2010), 81-101.

[9] N.M. Katz, On the monodromy groups attached to certain families of exponential sums, Duke Math. J. 54(1) (1987), 41-56.

[10] - Exponential sums and differential equations, Annals of Mathematics Studies, Vol. 124, Princeton University Press, Princeton, NJ (1990).

[11] P. Kurlberg and Z. Rudnick, The fluctuations in the number of points on a hyperelliptic curve over a finite field, J. Number Theory 129(3) (2009), 580-587.

[12] H.L. Montgomery, Ten lectures on the interface between analytic number theory and harmonic analysis, CBMS Regional Conference Series in Mathematics, Vol. 84, Amer. Math. Soc., Providence, RI (1994).

[13] R. Pries and H.J. Zhu, The p-rank stratification of Artin-Schreier curves, Ann. Inst. Fourier (Grenoble) 61(2) (2012), 707-726.

[14] A. Rojas-León and D. Wan, Improvements of the Weil bound for Artin-Schreier curves, Math. Ann. 351(2) (2011), 417-442.

[15] M. Rosen, Number theory in function fields, Graduate Texts in Mathematics, Vol. 210, SpringerVerlag, New York (2002). 
[16] M.M. Wood, The distribution of the number of points on trigonal curves over $\mathbb{F}_{q}$, Int. Math. Res. Not. IMRN (23) (2012), 5444-5456.

[17] M. Xiong, The fluctuations in the number of points on a family of curves over a finite field, J. Théor. Nombres Bordeaux 22(3) (2010), 755-769.

[18] - Statistics of the zeros of zeta functions in a family of curves over a finite field, Int. Math. Res. Not. IMRN (18) (2010), 3489-3518.

Department of Mathematics, University of California at San Diego, 9500 Gilman Drive NO. 0112, LA Jolla, CA 92093, USA

E-mail address: alina@math.ucsd.edu

Department of Mathematics and Statistics, Concordia University, 1455 de Maisonneuve West, Montreal, QC H3G 1M8, Canada

E-mail address: cdavid@mathstat.concordia.ca

Department of Mathematics, The City College of New York, CUNy, NAC 8/133, NEW YORK, NY 10031, USA

E-mail address: bfeigon@ccny.cuny.edu

DÉPARtement de mathématiques et de statistique, Université de Montréal, CP 6128, succ. Centre-ville, Montreal, QC H3C 3J7, Canada

E-mail address: mlalin@dms.umontreal.ca

Department of Mathematics, Indian Institute of Science Education and Research (IISER), First floor, Central Tower, Sai Trinity Bullding, Garware Circle, Sutarwadi, Pashan, Pune 411021, Maharashtra, India

E-mail address: kaneenika@gmail.com 